\title{
Transcriptional Regulation of Platelet Formation: Harnessing the Complexity for Efficient Platelet Production In Vitro
}

\author{
Marloes R. Tijssen, Thomas Moreau, and Cedric Ghevaert
}

\begin{abstract}
It is now common knowledge that specific repertoires of transcription factors (TFs) determine a cell's protein content and thereby its phenotype. The expression of a given TF is not necessarily cell specific, and many TFs play a pivotal role in several different cell types. For example, TAL1, FLI1, RUNX1, ERG and GATA2 are important regulators of stem cells, but also play a vital role in megakaryopoiesis. Although the megakaryocyte (MK) and its closest relative, the red blood cell, share key TFs like GATA1 and NFE2, the bifurcation between the two lineages has been associated with pairs of TFs that act as a toggle switch (such as FLI1 and KLF1). This chapter will summarise the current knowledge of key transcriptional regulators of MK differentiation and how some of these TFs, despite being expressed in several cell types, can impose MK cell identity. Since the discovery of TPO in 1994, our knowledge of MK biology and differentiation has increased exponentially, but we still lack a deep understanding of what triggers the transition from MK growth and maturation to proplatelet formation. We describe how some well-known TFs control the expression of proteins that play a pivotal role in the dramatic cytoplasmic and cytoskeletal events that accompany proplatelet formation. Finally, we show how TFs can be harnessed in a powerful way to produce MKs and, potentially, platelets in vitro for future clinical applications.
\end{abstract}

The original version of this chapter was revised. The copyright holder has been updated to The Author(s). An erratum to this chapter can be found at https://doi.org/10.1007/978-3-319-39562-3_21

M.R. Tijssen • T. Moreau • C. Ghevaert $(\varangle)$

Department of Haematology, NHS Blood and Transplant Centre, University of Cambridge,

Cambridge, UK

e-mail: mrt37@cam.ac.uk; tm384@medschl.cam.ac.uk; cg348@cam.ac.uk

H. Schulze, J. Italiano (eds.), Molecular and Cellular Biology of Platelet Formation,

DOI 10.1007/978-3-319-39562-3_2 


\subsection{Introduction}

Although the role of platelets clearly extends well beyond their primary role in homeostasis, the main symptom of thrombocytopenia (low platelet count) or platelet dysfunction is bleeding. Human peripheral blood contains $150-450 \times 10^{9}$ platelets $/ \mathrm{L}$, and since platelets have a life span of 10 days, there is a substantial demand for continuous platelet production in the bone marrow (estimated to be around $10^{11}$ platelets per day). This process is tightly regulated, and the body has means to increase it when more platelets are needed, for example, in the case of increased platelet consumption following a haemorrhage or infection. All blood cells originate from multipotent haematopoietic stem cells (HSCs) that can self-renew and differentiate into specialist cells. Platelets are derived from large polynucleated cells called the megakaryocytes (MKs) that are generated from HSCs. Although there is still a debate whether MKs descend directly from the HSCs, a specific population of stem cells, or go through the stage of a common myeloid progenitor first (see Sect. 2.2), we do have extensive knowledge on the transcription factors (TFs) involved in enforcing an MK cell fate. Once an MK has matured, it will ultimately release thousands of platelets into the bloodstream. Despite researchers rather efficiently recapitulating the process of MK formation and maturation from stem cells in the laboratory, we can at present only harvest five to ten functional platelets per MK in vitro. It is possible that these in vitro-derived MKs are not given the right environment to produce higher numbers of platelets or that the in vitro-produced platelets are somehow activated and therefore non-functional and lost from the final harvest. Alternatively, we may be missing crucial cues in vitro for the final step of MK maturation or platelet formation. Therefore, the knowledge of the transcriptional regulation of MK differentiation is extremely relevant in order to understand how platelets are produced and to ultimately harness that knowledge and translate it into the efficient in vitro production of platelets for clinical applications.

\subsection{Haematopoietic Stem Cell Differentiation: About Heterogeneity, Stochastic and Directed Fate Decisions}

The accepted central concept of "a stem cell" is that of a cell that possesses the ability to self-renew and is able to differentiate into lineage-restricted cells that become increasingly specialised until they reach the terminally differentiated fully mature cells. This concept was first demonstrated in the haematopoietic system in the $1950 \mathrm{~s}$ through mouse models of bone marrow transplantation. Low numbers of stem cells could repopulate the bone marrow of a mouse with a compromised haematopoietic system and regenerate all haematopoietic cell types. The multiple observations resulting from this large body of work have produced strong support for a hierarchical model in which the long-term self-renewal potential of the HSCs is restricted upon differentiation into lineage progenitors that will eventually produce the terminally differentiated mature blood and immune cells [1]. 
However, the advent of cell sorting technologies and single-cell assays has allowed us to realise that even a well-defined HSC population is heterogeneous, with each cell possessing specific differentiation patterns and self-renewal properties. Fascinatingly, these properties appear to be largely intrinsically stable within the original HSC and its progeny (reviewed in [2]), although external stimuli (usually described under the blanket term of "niche") can influence HSC behaviour (reviewed in [3]). This heterogeneity of HSC has been brought to light specifically in terms of MK differentiation by the demonstration by two independent groups of a previously undescribed subpopulation of HSCs which is "primed" to preferentially differentiate to the MK lineage $[4,5]$. This suggests HSCs do not necessarily need to go through the multipotent progenitor stage.

Despite a massive body of research into stem cell traits, the field is still divided with regard to the mechanism triggering the cell to stop self-renewing and to commit to a certain cell fate. There is obvious evidence for both a "directed" decision (i.e. under the influence of both external stimuli and intrinsic cell characteristics) and a stochastic process. However, a stochastic cell fate decision might only appear to be so as a consequence of our current inability to look into the minute molecular details of each individual cell in order to identify the probabilistic outcome of cell differentiation. The orchestration of the outcome of differentiation is ultimately driven at the cellular level by the precise arrangement of TFs in a given progenitor which will trigger the expression of lineage-specific gene regulatory networks.

This paradigm is illustrated by the bipotent MK-erythroid progenitors (MEPs) that can develop either into an erythroblast or an MK for which they need to switch on a completely different gene repertoire. Erythrocytes reduce their cell size and condense their nucleus, which is finally expelled. MKs on the other hand become bigger, increase their DNA content, and form specific "granules". Although the demonstration that pairs of TF "switches" may regulate the MEPs cell fate decision, the process by which the cell decides which TF to favour (and thereby the choice of pathway) is still unclear.

\subsection{Regulation of Gene Expression: Multilayer Complexity}

Each cell of the body contains the same genetic information, whilst its specific function and identity are defined by the restricted protein set that it contains and thereby its pattern of gene expression. Regardless of whether the cell fate decision is stochastic or directed, it will be executed by changes in the gene expression profile of the cell. The regulation of each protein concentration in a specific cell is subject to a very complex multilayered regulation at the transcriptional, translational and protein structure levels. This includes epigenetic regulation which relates to the threedimensional organisation of the chromatin and its accessibility to DNA-binding proteins that regulate gene transcription [6], non-coding RNA such as miRNA (which regulate degradation of mRNA or repress translation) [7] and long noncoding RNAs whose role in haematopoiesis (including erythroid/Mk maturation) is gradually being uncovered [8]. For example, although the level of TFs involved in 
late MK differentiation and platelet formation (such as GATA1, FOG1, FLI1, TAL1, RUNX1 and NFE2) does not increase with polyploidisation, the transcripts of their target genes are upregulated [9] indicating that several nonmutually exclusive mechanisms (translational and/or post-translational regulation) might be at play. In addition, a recent publication showed that an extra dimension is added to this transcriptional regulation by the lineage-specific expression of different isoforms from the same gene, conferring specific biological activity to the protein within a given cell type in the haematopoietic tree [10]. The mechanisms underlying lineagespecific alternative promoter use or splicing are still to be fully understood. Intriguingly, recent studies have shown that whilst devoid of nuclei, platelets are still endowed with gene expression control mechanisms including mRNA splicing in response to external cues and effective RNA interference machinery associated with miRNA expression $[11,12]$. This chapter will, however, concentrate specifically on the role of TFs in MK differentiation and, ultimately, platelet production.

Expression of actively transcribed genes is generally initiated by binding of ubiquitously expressed general TFs to the TATA sequence in the promoter of these genes. This leads to the recruitment of the transcription machinery consisting of other regulatory cofactors and RNA polymerases [13]. Specificity is introduced by the tight regulation of the expression of a repertoire of cell-type-specific TFs. These TFs recognise certain sequences in the DNA called motifs. These motifs are usually not long and appear many times in the DNA. Whether a TF will bind to its motif depends on the accessibility of the DNA and collaborative association with cofactors. To accommodate the large eukaryotic genome in the nucleus, the DNA is packed into chromatin. It is organised in nucleosomes by histone proteins and repeating units of nucleosomes make up the chromatin. The cell uses modifications of the histone proteins to regulate the tightness of the packaging and thereby DNA access to TFs and the transcriptional machinery. Interestingly, these modifications are inherited by daughter cells and are thus one of the ways to regulate cell-type specificity [14].

Complexes of different TFs can bind a single DNA element inducing transcriptional activation or repression depending on the constituents of the complex [15] (Fig. 2.1). The crucial role of TFs in lineage specification from HSC to MK and subsequent MK maturation is well documented [16], but the exact role that these TFs may play in enabling MKs to form platelets is not as clear. Some evidence can however be gleaned from existing data and will be discussed for individual key TFs in Sect. 2.5.

It was long thought that once a cell had gone down the path of differentiation, this decision was irreversible. However, as early cell nuclei transfer experiments suggested [17] and the birth of Dolly the sheep in 1996 spectacularly demonstrated, the transfer of a somatic nucleus into an egg can reverse programme the cell back into development to make it pluripotent. More recently, the discovery that this reversal of a cell's differentiation status (so-called reprogramming) can also be accomplished by overexpression of four TFs (thereby creating an "induced" pluripotent stem cell) earned Shinya Yamanaka a Nobel Prize [18]. It has become clear that overexpressing key TFs can not only reprogramme a somatic cell into a pluripotent cell, but that a somatic cell can be pushed into an alternative cell identity without necessarily going through the pluripotent stage (lateral programming) and that a similar approach can 

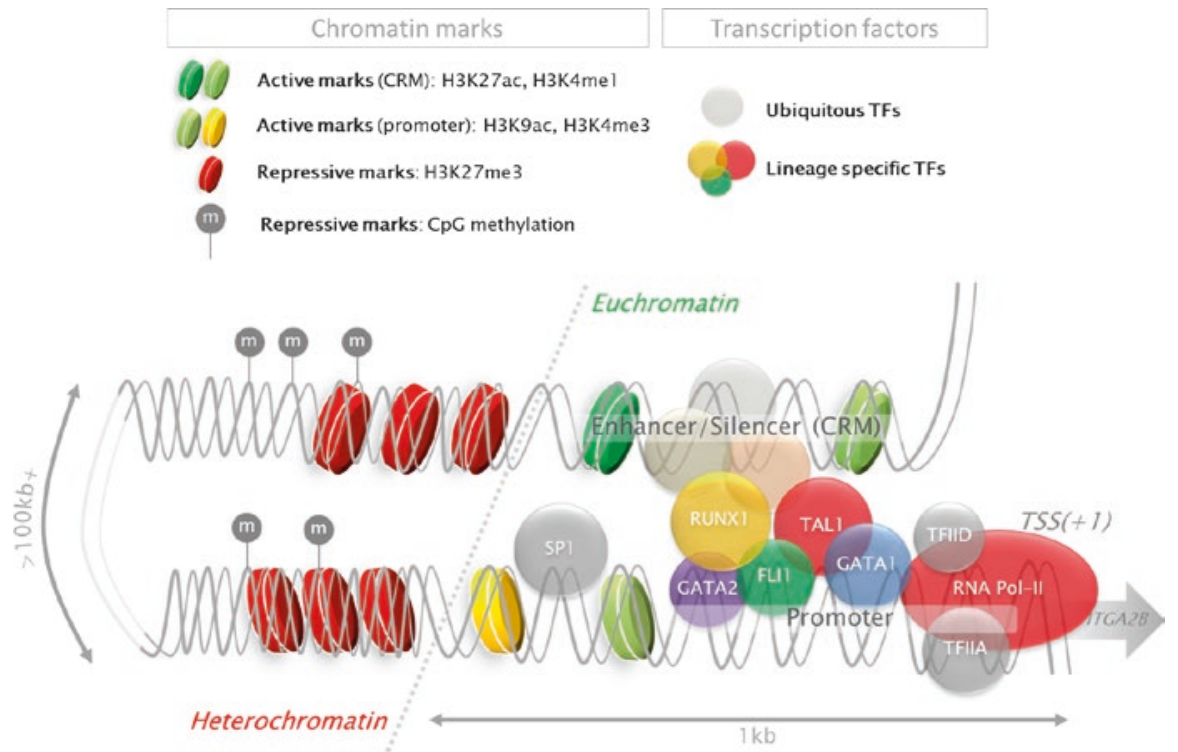

Fig. 2.1 Transcriptional regulation of gene expression. The specificity for spatio-temporal regulation of gene expression is acquired by combinatorial binding of multiple transcription factors (TFs). TFs and co-activators bind at the promoter of a gene proximal to the transcription start site (TSS). The subsequent recruitment of the RNA polymerase-II and ubiquitous transcriptional machinery factors will initiate transcription. Sets of TFs binding on a distal DNA element are called cis-regulatory modules (CRMs). CRMs can be located several hundreds of kilobases (kb) or even more away from the promoter and still function as enhancers/repressors of gene expression through long-range DNA loop interaction. Gene expression is further regulated by the chromatin structure (opened euchromatin and closed heterochromatin) guided by epigenetic modifications involving histone modifications (e.g. acetylation and methylation of lysine residues on $\mathrm{H} 3$ ) and DNA methylation on cytosine which ultimately controls accessibility of TFs to their DNA-binding sequence. These mechanisms are illustrated here for the key megakaryocyte surface receptor gene integrin alpha-2b (ITGA2B) based on ChIP-Seq data [206, 244]

force a pluripotent stem cell towards a specific stem cell fate (forward programming). The latter has great potential for the generation of cells from patients (enabling disease modelling) or the production of cells for clinical use and will also be discussed in the context of megakaryopoiesis and in vitro platelet production.

\subsection{Making Platelets: The Megakaryocyte, Its Niche and Its DNA Content}

\subsubsection{MK Specification and Differentiation}

The main driver of MK differentiation is the binding of thrombopoietin (TPO) to its receptor MPL [19]. The subsequent dimerisation of the receptor induces the autophosphorylation of the Janus kinase 2 (JAK2). JAK2 phosphorylates a number of 
downstream substrates leading to the activation of multiple signalling pathways including mitogen-activated protein kinases (MAPK), phosphoinositol-3 kinase (PI3K) and signal transducers and activators of transcription (STATs). The ultimate effect of the activation of these signalling pathways is induction and repression of gene expression and MK differentiation.

Besides TPO, other cytokines, chemokines and extracellular matrix proteins influence megakaryopoiesis [20,21]. Noteworthy is the residual level of platelets (about 15\%) in Tpo or $\mathrm{Mpl}$ knockout mice probably driven by the interaction between the MK progenitor and the BM endothelial niche [22].

\subsubsection{Triggers for Proplatelet Formation and Platelet Release: The Little Bit We Do Know}

As the process of platelet formation is still debated, so is the trigger for this process. Although the role of TPO in MK maturation is undisputed, TPO seems dispensable for platelet formation and maybe even inhibits this process [23, 24]. Removal of growth factors commonly triggers cell death. Therefore, the dispensability of TPO may be related to the fact that proplatelet formation has been described as a form of compartmentalised caspase-dependent cell death. Overexpression of antiapoptotic molecules (BCL2 and BCL2L1 [BclxL]) or reduced expression of proapoptotic members of the BCL2 family (BCL2L11 [Bim]) reduces platelet formation by MKs [2527] as does pharmacological inhibition of caspases [28, 29]. A recent study confirmed the central role played by BCL2L1 in platelet release from mature MKs, although in this particular study, mice with combined deletion of both proapoptotic BAK1 and BAX proteins showed no alteration of platelet formation [30]. Also, a lack of caspase-9 or BCL2, in contrast to overexpression, does not seem to affect steady-state platelet formation $[31,32]$. Together, these studies suggest that proplatelet formation does not require the activation of the intrinsic (mitochondrial) apoptosis pathway.

The location of the MK and interaction with its environment seem to be crucial for platelet formation. It is known that the relocation of immature MKs from the osteoblastic niche to the endothelial niche driven by chemotactic agents is essential for maturation and platelet production [21]. Contact with bone marrow endothelial cells (BMECs) induces further maturation and platelet formation [33]. The chemokine CXCL12 (SDF1 $\alpha$ ) and growth factor FGF4 promote both MK migration and interaction with the BMECs, thereby promoting platelet production [22]. Interestingly, this interaction is enhanced by inflammatory cytokines, such as interleukin-1 beta (IL-1B) [33, 34]. Furthermore, stimulation of the pro-inflammatory VEGFR1 pathway leads to an upregulation of the CXCL12 receptor CXCR4 and increased in vivo platelet formation [35]. These findings suggest that increased interaction between MKs and stimulated endothelium is responsible for the thrombocytosis often observed in inflammation [36]. It is also clear that when MK migration is impaired, platelet formation is impaired too. This is true for Wiskott-Aldrich syndrome (WAS) where actin polymerisation is disturbed [37] and for thrombocytopenia induced by compounds like the kinase inhibitor dasatinib that decrease MK migration [38]. 
In addition to this direct MK-to-endothelial cell contact, extracellular matrix proteins influence proplatelet formation. Type I collagen inhibits proplatelet formation through integrin $\alpha$ III $\beta$ I [39]. Fibrinogen on the other hand promotes proplatelet formation through interaction with glycoprotein IIB/IIIA (GPIIB/IIIA, integrin $\alpha I I b \beta 3)$. Mutations causing constitutive GPIIB/IIIA activation interfere with proplatelet formation and lead to the production of very large platelets (macrothrombocytopenia) [40, 41]. There is also evidence that von Willebrand factor (vWF) binding to the GPIB/V/IX complex regulates proplatelet formation, which may be the reason for the macrothrombocytopenia observed in patients with Bernard-Soulier syndrome who suffer from a defect in the GPIB/V/IX complex [42]. Platelet production seems therefore under control of several adhesive interactions between the MK, the $\mathrm{BM}$ vasculature and the extracellular matrix. However, these do not trigger the protrusion of the proplatelet extensions into the sinusoids. For this, one would expect the presence of a compelling factor in the serum, which could be sphingosine 1-phosphate [43, 44].

\subsubsection{MK DNA Content: Does Ploidy Really Matter?}

Maturation of MKs is accompanied by successive rounds of DNA replication without cytokinesis (endomitosis), resulting in large polyploid cells with a lobulated nucleus. It has been hypothesised that polyploidisation is required to meet the MK's vast need of protein synthesis and cell growth, as well as production of the demarcation membrane system which provides extra cell membrane supply for platelet formation [45]. This is supported by a study by Raslova et al. showing that all alleles of a series of MK-specific genes in cells ranging from $4 \mathrm{~N}$ to $32 \mathrm{~N}$ are functional and not epigenetically silenced [9]. However, a subsequent study, by the same authors, showed that multiple genes involved in platelet formation and DNA proliferation are regulated differently depending on ploidy levels [46]. Thus, specific gene regulatory processes are at work at different levels of ploidy to ensure the MK develops into a platelet-producing cell. Indeed, murine MKs with high ploidy have downregulated genes involved in DNA replication and upregulated genes involved in cytoskeletal dynamics, cell migration, G-protein signalling and platelet function [47].

Surprisingly, proplatelet formation is not strictly within the remit of the higher ploidy MKs. Both in vivo and in culture 2N or 4N MKs form proplatelets [48-50], and platelet numbers are similar in mouse backgrounds with different levels of BM MK polyploidy [51]. Therefore, although some cytoskeletal (TUBB1, MYH9, RAC1, RAP1B), transmembrane (GPIIIA, GP1BA and B) and signalling proteins (LAT and SRC family kinases) known to be involved in proplatelet formation are slightly more abundant in high-ploidy MKs, this appears not to affect platelet shedding. However, instead of having an effect on its ability to produce platelets, the ploidy level of an individual MK might influence protein content. Platelets originating from high-ploidy MKs might be more easily activated than platelets generated from MKs with a lower ploidy [52]. 
In keeping with the idea that platelet shedding is not necessarily coupled with ploidy, genes regulating platelet production, such as nuclear factor erythroidderived 2 (NFE2, discussed below), are not involved in the regulation of polyploidisation [53], and inversely, genes that increase MK ploidy, such as cyclin D3 (CCND3), do not modify the platelet count [54].

\subsection{Transcription Factors Controlling the Megakaryocyte Cell Fate}

\subsubsection{The GATAs}

\subsubsection{GATA1 and GATA2}

The six members of the GATA (globin transcription factor) family of TFs recognise [5'-(A/T)GATA(A/G)-3'] motifs for which they are named [55]. The defining feature of this family is the presence of two highly conserved Cys4 zinc fingers. The C-terminal finger binds DNA, whilst the N-terminal finger mediates the binding of cofactors [56] and may be involved in stabilising DNA binding [57]. The GATA $\mathrm{N}$-terminal domain regulates target genes depending on the context of the cell type through its binding to co-activators. Interestingly, genes that have low sensitivity to GATA activation are more prominently affected by mutations affecting binding to co-activators [58].

GATA1-3 are known as the haematopoietic GATA factors although they have also been found to be expressed in other tissues. GATA3 is expressed in HSCs and lymphoid cells, but not in MKs. Both GATA1 and GATA2 are expressed in the MK lineage, erythroid progenitors, eosinophils and mast cells. Importantly, GATA factors are also endowed with pioneer activity - i.e. they can access their DNA target sites in compact chromatin and presumably bind to the genome before the binding of other factors, a phenomenon notably at play in the process of lineage anticipation [59].

GATA2 is essential for the appearance of early haematopoietic progenitor cells [60]. It is part of the "stem cell heptad" (together with TAL1, LYL1, LMO2, RUNX1, FLI1 and ERG) defined by overlapping occupancy in chromatin immunoprecipitation (ChIP) experiments combined with deep whole genome sequencing (ChIP-Seq) in the mouse haematopoietic progenitor cell-7 (HPC-7) cell line [61]. GATA2 is necessary for the early commitment to the red cell lineage. Subsequent induction of GATA1 expression in the red cell lineage leads to a rapid decrease of expression of GATA2 (the "GATA switch") which is necessary to achieve terminal red cell maturation [62]. In the red cell lineage, GATA1binding sites appear "de novo" in the maturing erythroblasts, whilst in MKs, $40 \%$ of GATA1-binding sites correspond to sites already occupied by GATA2 in HSCs. These sites are enriched for TAL1 and strongly associated with gene induction [63]. Unlike the red cell lineage, GATA2 expression is maintained alongside GATA1 in the developing MKs with evidence that GATA2 can compensate and reinforce MK identity in the absence of GATA1 [64, 65]. The 
GATA1 - and GATA2-bound sites in mature MKs do not overlap, however, with GATA1 sites found mostly in platelet-specific genes (and enriched for ETS factor motifs) and GATA2 sites corresponding to genes expressed in stem cells that are repressed in MKs [63].

GATA1 is expressed primarily in mature cell types, including red cells and MKs. GATA1 deficiency leads to a complete block of erythroid maturation, but megakaryopoiesis is not completely absent, presumably due to some compensation by overexpression of GATA2. However, GATA1 deficiency leads to a decrease in ploidy and abnormalities of certain features that are characteristic of MK terminal maturation (such as cytoplasmic granules) $[66,67]$. Overexpression of some downstream targets of GATA1 can restore, at least in part, some of the MK phenotype: cyclin D1 (CCND1) overexpression increases ploidy, but not cytoplasmic maturation [68], whereas overexpression of signal transducer and activator of transcription 1 (STAT1) or IFN regulatory factor-1 (IFR1, its downstream effector) not only increases DNA content but also expression of platelet-specific genes.

A key role for the regulation of MK-specific genes expression by GATA1 is illustrated by human pathologies: for example, a point mutation in a GATA-binding site in the glycoprotein $1 \mathrm{~B} \beta$ subunit $(G P 1 B B ; C D 42 b)$ promoter proximal region causes a form of Bernard-Soulier syndrome [69].

The GATA1 gene locus is on the X chromosome (Xp11.23). X-linked mutations of GATAl present with a wide range of phenotypes. Patients have bleeding tendencies ranging in severity, associated in some instances, with red cell abnormalities. These patients show not only a decrease in the number of platelets, but their platelets also lack key features such as alpha granules [70]. The variety of phenotypes observed with GATA1 mutation relates to whether the mutation affects binding of GATA1 to the DNA itself or to cofactors such as FOG1 [71] and TAL1 [72].

\subsubsection{FOG1 and Other Partners of GATA1}

Friend of GATA1 (FOG1, encoded by the gene ZFPM1 on chromosome 16q24.2) does not bind to DNA, but binds to GATA1 and GATA2. It was originally identified as a GATA1 co-partner in yeast two-hybrid studies and shown to be a coactivator of transcription for haematopoietic genes such as NFE2 and to play a role in both erythroid and MK development [56]. This was confirmed in Fog 1 knockout mice that die at mid-gestation with severe anaemia and absence of platelet production [73]. The loss of FOG1 leads to a decrease of GATA1 binding to its DNA targets and, crucially, prevents the association of the GATA1/FOG1 complex with the nucleosome remodelling and deacetylase (NURD) complex. The formation of the "GATA1/FOG1/NURD" complex leads to the efficient regulation of gene transcription necessary to enforce the lineage commitment and cell maturation [74, 75]. Loss of Fogl in a conditional Mx-Cre mouse model leads to a much more extensive phenotype than the loss of Gatal using the same Mx-Cre conditional approach. This is due to a deficient repression of Gata 2 by GATA1 and a complete loss of erythroid/MK commitment in progenitors and a skewing towards myeloid differentiation [76, 77]. Interestingly FOG1 can also inhibit 
GATA1-driven transcription at certain promoter sites, which is mediated by binding of FOG1 to the corepressor C-terminal binding protein 2 (CTBP2) [78]. Overexpressing either wild-type GATA1 or a mutant GATA1V205G (which does not bind FOG1) in an erythroid-MK cell line deficient in endogenous GATA1 (G1ME), Chlon et al. showed that GATA1V205G bound to genomic regions distinct from those bound by wild-type GATA1. In particular, in the absence of FOG1, wild-type GATA1 was less likely to bind to promoter regions, more likely to bind into regions of "closed chromatin" and less likely to bind to regions where concurrent ETS factor (see Sect. 2.5.4)-binding motifs are found. Crucially, overexpression of GATA1V205G failed to induce transcription of MK genes, but instead led to overexpression of genes belonging to the myeloid lineage (particularly mast cell genes). Therefore, binding of GATA1 to its cofactor FOG1 maintains erythroid/MK lineage fidelity [79].

In addition to FOG1, GATA1 combines with other TFs such as leukaemia/ lymphoma-related factor (LRF, encoded by ZBTB7A), TAL1 and CBFA2T3 (corebinding factor, runt domain, alpha subunit 2; translocated to 3 , also known as ETO2). Interestingly, the association of TAL1 with GATA1 is only seen at genomic loci where GATA1 acts as an activator not as a repressor [75]. CBFA2T3 acts as a corepressor of GATA1-bound genes. It is only expressed in immature MKs where it presumably represses inappropriate early expression of a subset of genes expressed in terminally differentiated MKs [80], and therefore Cbfa2t3 knockdown promotes MK maturation. ChIP-Seq studies have been carried out in primary murine cells in order to look specifically at the erythroid/MK lineage bifurcation and have shown some key features of GATA1 association with other TFs. GATA1-binding sites in MKs are mostly distinct to those seen in erythroblasts. The genomic region around these GATA1-binding sites is enriched for ETS and RUNX motifs in the former, whilst they are enriched for KLF1-binding motifs in the latter. Whilst clearly the combination of GATA1 and FLI1 binding identifies genes that are actively transcribed in MKs, the combination of GATA1 and ETS factor elements results in gene silencing in the erythroid lineage [63].

\subsubsection{Down Syndrome and Megakaryopoiesis}

Foetuses with trisomy 21 (Down syndrome) have haematological abnormalities characterised by an expansion of MK-erythroid progenitors in the foetal liver during the second trimester [81]. This expansion is dramatically increased in 5\% of children with Down syndrome and develops into a transient clonal myeloproliferative disorder (TMD) characterised by an expansion of immature MKs which often undergoes spontaneous remission. This is the result of an acquired mutation on the $\mathrm{X}$ chromosome resulting in the production of a shorter isoform of GATA1, so-called GATA1s [82]. GATA1s lack the N-terminal transactivation domain and is normally co-expressed with full-length GATA1 [83]. Mice that express Gatals only show MK proliferation with maturation arrest in the prenatal period [84], which is thought to be driven by the removal of the inhibitory activity of the GATA1 N-terminal domain on E2F [85]. Down syndrome patients can develop a specific type of childhood megakaryocytic leukaemia (DS AMKL) that has a much better prognosis than 
other forms of AMKL. It is thought that it is the combination of GATA1s with overexpression of other genes (such as $E R G$, see Sect. 2.5.4.2) through the trisomy 21 that leads to DS AMKL.

\subsubsection{TAL1}

T-cell acute lymphocytic leukaemia 1 (TAL1, also called as SCL) is a basic-helixloop-helix (bHLH) TF (encoded on chromosome 1p32). TAL1 functions as an obligate heterodimer with the ubiquitously expressed bHLH TF E2A. It binds to its consensus sequence, the so-called E-box (CANNTG). In red cells it functions in a multiprotein complex, which also includes LMO2 (LIM domain only 2) and LIM domain-binding 1 (LDB1). Depending on other factors recruited to this complex (such as EP300, GFI1B, CBFA2T3 and KDM1A), it can function as an activator or repressor of transcription (summarised in [86]). As mentioned above, TAL1 binding in conjunction with GATA1 distinguishes active from repressed transcription sites. Furthermore, evolutionary conserved association of E-box and GATA motifs separated by 9-12 base pairs has been documented in regulatory regions of several erythroid/MK genes including GATA1 itself [67]. A study of TAL1-binding sites in primary mouse erythroid and MK cells has shown very distinctive patterns of binding, which is conferred by the state of the chromatin (active/inactive) and interactions with other TFs, particularly GATA1. The phenomenon of lineage anticipation was also clearly demonstrated in this study, with a significant number of MK genes bound by TAL1 in mature MKs, already bound by TAL1 in the HPC-7 haematopoietic progenitor cell line [87].

TAL1 is essential for the emergence of all haematopoietic lineages, which has been demonstrated both in vivo (Tall knockout mice die between embryonic day 8.5-10.5 due to a complete lack of blood formation) [88] and in vitro [89]. Although TAL1 is necessary for the emergence of HSCs, the use of Mx-Cre and Tie2-Cre conditional models has shown that TAL1 is dispensable for the maintenance of long-term repopulating stem cells and their multipotency, but is absolutely required for differentiation of the erythro-MK lineage [90, 91]. Knockdown of TAL1 translates into a lack of proliferation, polyploidisation and cytoplasmic maturation of the MKs and a reduced platelet number [92]. One of the targets of TAL1 is the cellcycle regulator cyclin-dependent kinase inhibitor 1A (CDKN1A, p21) which is overexpressed upon knockdown of TAL1 expression [92]. Crucially, knockdown of CDKN1A in TAL1-mutant MKs restores the endomitotic cell-cycle progression, but only partially restores the cytoplasmic maturation necessary for the production of fully functional platelets. Thus, other targets of TAL1 are also responsible for the defects in these late stages of MK maturation. One of these targets may be myocyte enhancer factor $2 \mathrm{C}$ (MEF2C), as mice lacking $\mathrm{MEF} 2 \mathrm{C}$ in the haematopoietic lineage have reduced numbers of platelets with larger size and abnormal shape and granularity [93].

Other TAL1-interacting proteins include epigenetic modifier enzymes such as LSD1 (histone 3 lysine 4 demethylase), HDAC1 and HDAC2 (histone deacetylases) 
and the corepressor molecule CoREST [94]. CoREST (REST corepressor 1, encoded by the RCORI gene) was recently identified as part of a genome-wide association study as a locus linked to platelet count in humans [95], and its role in haematopoietic lineage specification has been recently confirmed in a zebrafish model [96].

\subsubsection{RUNX1}

\subsubsection{RUNX1 and Haematopoiesis}

RUNX1 (runt-related transcription factor 1, aka AML1) is a member of the RUNT family of TFs and together with its cofactor core-binding factor, beta subunit (CBFB ) represents the most common mutational target in human acute leukaemia. RUNX1 plays an essential role in definitive HSC emergence from the aorta-gonadmesonephros region during embryogenesis [97]. Therefore complete absence of RUNX1 in mice is embryonic lethal [98]. However, using a murine Mx-Cre conditional system which allows the deletion of Runxl from adult haematopoiesis, a clear role for RUNX1 was shown for the development of lymphocytes and MKs, whilst myelopoiesis and HSC function were preserved [99], similar to what is observed for TAL1. Runxl deletion resulted in the accumulation of small immature MK progenitors within the bone marrow and a marked decrease in polyploidisation and cytoplasmic development of MKs, similarly to what is observed for GATA1 deficiency.

\subsubsection{Familial Platelet Disorder/AML}

The complex role of RUNX1 in MK differentiation is illustrated by the autosomal dominant human syndrome familial platelet disorder with propensity to develop acute myeloid leukaemia (FDP/AML) in which germline heterozygous RUNXI mutations lead not only to thrombocytopenia but also to impaired platelet function, in addition to a high risk for the development of myelodysplasia and leukaemia [100]. In MKs cultured from FPD/AML patients' stem cells, expression of non-muscle myosin is perturbed with persistent expression of MYH10 (non-muscle myosin IIb) and decreased expression of MYL9 and MYH9 (non-muscle myosin IIa) [101, 102]. Actin and myosin control branching of the proplatelet elongations and MYH9 alongside its regulator RHOA (RAS homolog family member A) restrain platelet formation. Consequently, mutations that reduce myosin IIa activity such as seen in the MYH9-related May-Hegglin syndrome lead to inappropriate platelet shedding causing macrothrombocytopenia [103, 104]. MYH10 specifically localises to the contractile ring separating the cell during mitosis, and its silencing by RUNX1 contributes to the transition from mitosis to endomitosis as the MKs mature [105]. Thus, RUNX1 regulates key constituents of the MK and platelet cytoskeleton and thereby affects features of late megakaryopoiesis such as polyploidisation and platelet formation. 


\subsubsection{RUNX1 in Partnership}

The role of RUNX1 in megakaryopoiesis is a perfect illustration of how TFs are part of a network of regulatory proteins with differential effects depending on the cellular stage and crucially other TF partners. One publication analysed the dynamics of gene expression regulation during megakaryopoiesis using three cell line models: the erythroleukaemia K562 cell line, K562 cells induced towards the MK lineage using 12-O-tetradecanoylphorbol-13-acetate (TPA) and the megakaryoblastic CMK cell line, which reportedly represents a more mature MK type than the K562 cell line. Although the results have to be interpreted with caution due to the use of cell lines, the authors report that constitutive RUNX1-binding sites (i.e. present in K562 cells prior to induction) were enriched for GATA1 motifs, whilst in the TPA-induced K562, de novo RUNX1-binding sites regulating the expression of MK-specific genes were enriched for the AP1 complex binding motif. In the CMK line, looking at genes more specifically expressed in the later stages of MK differentiation (such as RAB27B), the authors showed enrichment for the ETS binding motif. They therefore proposed a model whereby RUNX1 controls early MK differentiation genes in collaboration with GATA1, whilst for genes which are more typically expressed in later stages, RUNX1 regulation is effected in collaboration with FOS/ JUN family members and ETS TFs [106]. Another example of how RUNX1 can regulate the expression of the same gene, but with different partners, is exemplified by the analysis of the promoter for myeloproliferative leukaemia ( $M P L$, virus oncogene, the TPO receptor) where RUNX1 interacts with the SIN3A corepressor complex in haematopoietic stem and progenitor cells, whilst it forms a complex with the transcription activator EP300 (E1A-binding protein p300) on the same promoter in MKs [107]. The co-occupancy of RUNX1 and EP300 on the promoter region of multiple key MK genes was later confirmed in a genome-wide ChIP-Seq study using primary murine MKs [108].

\subsubsection{ETS Factors}

\subsubsection{FLI1}

The E26 transformation-specific (ETS) family of TFs contains more than 20 helixloop-helix domain TFs. Friend leukaemia virus integration 1 (FLI1, encoded on chromosome 11q24) is involved in several types of malignancies such as Ewing's sarcoma in humans [109] and erythroleukaemia in mice [110]. Evidence for its contribution to haematopoiesis comes from knockout mice. These mice die at day 11.5 of embryogenesis of cerebral bleeds caused by a lack of vascular integrity. In those animals, megakaryopoiesis was clearly affected with an increase in MK forming colonies, accompanied by the production of small MKs that lack expression of maturity markers [111]. Forced expression of FLI1 induces MK differentiation in K562 cells [112], and the overexpressed FLI1 binds to MK-specific gene promoters such as the promoter of ITGA2B (GPIIB, see Sect. 2.4.2) where it acts in synergy with GATA1 and FOG1 [113]. Inducible deletion of FLI1 in the 
haematopoietic compartment showed a marked increase in bipotent MEPs, which, in colony assays, were biased towards the erythroid compartment [114]. This observation is consistent with the concept that FLI1 and EKLF act as a toggle switch in MEPs each favouring differentiation towards the MK or erythroid lineage, respectively [115].

A lack of FLI1, however, does not seem to affect early commitment to the MK lineage as the number of ITGA2B-positive early MKs was only marginally decreased in knockdown studies [111]. It has in fact been shown that FLI1 acts in tandem with another ETS family TF GABPA (GA-binding protein TF alpha subunit). GAPBA is composed of a DNA-binding $\alpha$-subunit and a $\beta$-subunit responsible for nuclear localisation. As MK maturation progresses, the ratio of FLI1/GABPA increases. In keeping with this observation, GABPA regulates expression of early MK genes (including $I T G A 2 B$ and $M P L$ ), whilst FLI1 binds to both early and late (GPIBA, GP9 [both part of the von Willebrand factor receptor complex GPIb-V-IX] and PF4 [platelet factor 4]) MK genes [116].

The key role of FLI1 in the terminal differentiation of MKs is illustrated in the Paris-Trousseau syndrome, an inherited disorder associated with an 11q chromosome deletion with thrombocytopenia and an increased tendency to bleed. Hemizygous loss of FLI1 due to the deletion underlies the disease [117]. Patients show a maturation block with microMKs on BM smears and typical abnormal granule formation $[117,118]$.

\subsubsection{ERG}

ERG (v-ets avian erythroblastosis virus E26 oncogene homolog) is another member of the ETS protein family and is the most closely related to FLI1. In the multipotent murine HPC-7 line, ERG genome-wide occupancy paralleled that of FLI1 [61]. Studies of ERG mutant mice have shown that it is essential for the establishment of definitive haematopoiesis and stem cell maintenance as well as production of platelets [119]. ERG is a well-known oncogene: its expression level correlates with bad prognosis in myeloid leukaemias. In murine stem cells, ERG overexpression leads to both lymphoid leukaemia and myeloid leukaemia with erythromegakaryocytic characteristics [120,121].

ERG is encoded by a gene on chromosome 21q22.3 and has been implicated in the development of DS AMKL. In a mouse model, overexpressed ERG was shown to synergise with GATA1s to potentiate the expansion of foetal MK-erythroid progenitors [122]. These mice went on to develop frank leukaemia by 3 months of age. In a separate study, $\mathrm{Erg}$ overexpression was found to immortalise haematopoietic stem cells expressing Gatals, but not full-length Gatal [123]. Interestingly ChIPSeq profiling in haematopoietic cells overexpressing Erg identified potential therapeutic targets such as the PIM1 kinase. PIM1 inhibition could potentially be used to treat leukaemias when the leukaemic blasts overexpress $E R G$ [121]. $A K T$ is overexpressed in DS AMKL, and it has been recently shown that AKT-induced MK apoptosis is inhibited by both ERG and GATA1s and that in fact GATA1s block MK differentiation induced by AKT and sustain long-term proliferation, which makes the AKT pathway another potential target for therapy in DS AMKL [124]. 


\subsubsection{ETV6}

ETS variant 6 (ETV6, also called TEL) is another ETS family TF which is frequently rearranged and fused to other partners in chromosomal translocations seen in leukaemia. Although Tel knockout mice die of vascular malformations by day 11 post-fertilisation, blood formation in the embryo is largely unaffected [125]. However, in an inducible $\mathrm{Tel}$ knockout mouse model, deletion of $\mathrm{Tel}$ in the stem cell compartment using Mx-Cre resulted in a dramatic decrease of the HSCs content in the bone marrow, with a gradual recovery from the cells where the floxed allele was not excised. This indicates an absolute requirement for TEL in the maintenance of adult haematopoietic stem cells. Noticeably, following Mx-Cre induction, mature blood cells were unaffected except for a dramatic drop in the platelet count. In keeping with this observation, excision of $\mathrm{Tel}$ in the MK-erythroid compartment using Cre expression driven by the Gatal promoter had no effect on red cell production, but again caused a drop in the platelet count $>50 \%$. This was accompanied by an increase in MK forming colonies, suggesting that TEL plays a role in MK maturation rather than lineage commitment [126].

\subsubsection{EVI1}

Ecotropic virus integration site 1 (EVI1) is a member of the SET/PR domain family of TFs, and it contains a total of ten zinc finger motifs organised in two discrete domains, located at the $\mathrm{N}$ terminus and towards the $\mathrm{C}$ terminus, comprising seven (ZF1) and three (ZF2) repeats, respectively, which have distinct DNA-binding specificities [127].

The first evidence that EVI1 plays a role in megakaryopoiesis comes from patients with myeloid leukaemias. The MECOM locus on chromosome 3, encoding EVI1, is implicated in translocations in 4-6\% of all AML cases. These so-called 3q21q26 syndrome leukaemias present with particular dysmorphic MKs and an elevated platelet count. This is thought to be caused by inhibition of cyclin A-dependent kinase inhibitor 2 (CDK2) expression mediated by the abnormal expression of EVI1 [128]. EVI1 is expressed in haematopoietic progenitor cells and MKs. Cell line experiments have shown that EVI1 regulates expression of MK-specific genes. Ectopic expression in the human megakaryoblastic UT-7/GM cell line changes these cells into polynuclear large cells that express PF4 [129]. Knockdown of EVI1 in K562 cells reduces ITGA2B and ITGB3 expression after TPA treatment [130].

The evidence is further strengthened by animal models. Evil knockout mice die by day 10.5 of gestation due to widespread hypocellularity of the paraxial mesenchyme and haemorrhages. Conditional knockdown in Evil Mx-Cre mice has shown that EVI1 is essential for both embryonic and adult stem cell maintenance and repopulating activity. Lineage commitment appears conserved, but after Cre activation the mice developed mild thrombocytopenia and showed delayed platelet recovery after 5FU administration [131]. The role of EVI in stem cell (and MK) biology may be mediated through the expression of GATA2 and RUNX1, which are both regulated by EVI1 [132, 133]. 
Further evidence that EVI1 transcriptional regulation plays a key role in megakaryopoiesis comes from whole exome sequencing showing that a mutation creating an EVI1-binding site in the promoter of the RNA-binding motif protein 8A (RBM8A) gene underlies the thrombocytopenia with absent radii (TAR) syndrome [134]. In $80 \%$ of a cohort of TAR patients, a single nucleotide polymorphism (SNP) was discovered in the 5'UTR of RBM8A. The SNP increases binding of EVI1 and leads to a reduction of transcription of $R B M 8 A$ and the encoded protein Y14. TAR patients have low numbers of MKs in the BM, which seem to have a maturation defect [135]. In addition there is some evidence that platelet function may be abnormal in these patients [136-138]. Therefore, one might speculate that EVI1 regulation of $R B M 8 A$ is crucial for late MK differentiation.

\subsubsection{NFE2 and Related Transcription Factors}

\subsubsection{NFE2}

NFE2 was first identified as a transcriptional regulator in erythroblasts where it binds to the $\beta$-globin locus in a region that contains the core symmetric AP- 1 motif TCAT/C to which dimers of JUN and FOS subfamily proteins are bound. NFE2 is an obligate dimer between a large $45 \mathrm{KDa}$ subunit specifically expressed in haematopoietic cells (p45 NFE2, encoded on chromosome 12) and a choice of smaller, widely expressed, $18 \mathrm{KDa}$ subunits belonging to the MAF family (p18). MAFK is the prominent $\mathrm{p} 18$ expressed in erythroblasts, whilst $M A F G$ and $M A F F$ predominate in MKs, which may dictate the binding site preference on DNA sequences and/ or p45 NFE2 activity [139]. Remarkably p45 Nfe2 knockout mice display a mild dyserythropoiesis, but completely lack circulating platelets. This is despite an apparent increased number of MKs in the BM [53]. It was therefore proposed that NFE2 acts as a regulator of proplatelet formation by promoting the final stage of maturation of MKs to the point where they are capable of platelet shedding. NFE2deficient MKs can be grown in vitro in response to TPO, but are unusually large, and have a disorganised demarcation membrane and granules which are small and sparse indicating a late maturation block [140]. Knocking out MafG phenocopies the findings in the p45 Nfe 2 knockout mice, confirming its specific role in megakaryopoiesis [141].

Overexpression of NFE2 has been reported in patients with all three subtypes of myeloproliferative neoplasms, independent of the presence or absence of the JAK2V617F mutation [142, 143]. When expressed ectopically in BM cells, NFE2 also influences earlier stages of MK differentiation and allegedly enhances in vivo platelet production. However, transplantation of NFE2 overexpressing cells only accelerated platelet production and did not lead to an increase in the maximum level or total number of platelets detected in recipient blood [144].

The apparent role of NFE2 in proplatelet formation might be explained through the function of some of its direct transcriptional targets such as tubulin $\beta 1$ (TUBB1) [145], RAB27b [146], caspase 12 (CASP12) [147] and HSD3B1 (3-betahydroxysteroid dehydrogenase) [148]. Tubbl knockout mice have thrombocytopenia 
with spherical platelets [149], and a mutation in TUBB1 has been identified in a patient with congenital macrothrombocytopenia [150]. Mice with deficient RAB signalling have macrothrombocytopenia with few granules and abnormal MK morphology, and RAB27B may coordinate granule transport during proplatelet formation [146]. Nfe2 null MKs fail to bind to fibrinogen in response to platelet agonists indicative of a defect in the signalling leading to activation of GPIIB/IIIA, and, similarly, Casp12 null platelets have a defect in GPIIB/IIIA which is a well-documented positive regulator of proplatelet formation [151]. Finally, HSD3B1 (an enzyme implicated in oestrogen metabolism) rescues proplatelet formation in $\mathrm{Nfe} 2$ null MKs [148]. The authors conclude that MKs may secrete autocrine estradiol that regulates proplatelet formation. Additional studies have shown that oestrogens can induce MK differentiation [152, 153], but to our knowledge this has not been successfully applied to increase in vitro platelet formation.

\subsubsection{BACH1 and BACH2}

The NFE2 heterodimer binds to MARE (musculoaponeurotic fibrosarcoma oncogene recognition), a cis-regulatory element that can be bound by a variety of heterodimers consisting of MAF and p45-related proteins such as BACH1 (BTB and $\mathrm{CNC}$ homology 1) and $\mathrm{BACH} 2$, both of which are repressors of transcription. $B A C H 1$ (encoded on chromosome 21q22) is expressed in both the erythroid and MK compartment. In murine models, a lack of BACH1 does not affect platelet counts, but when Bachl is overexpressed specifically in the erythroblasts and MKs using the Gatal promoter, the mice showed thrombocytopenia, very similar to the $N f e 2$-deficient mice (including a defect in proplatelet formation), but without an effect on erythroid differentiation [154]. In this particular study, the authors showed that target genes of NFE2 (such as thromboxane A2 synthetase) were bound by BACH1 and their levels of expression reduced. Overexpression studies are difficult to interpret, but the authors propose that relative levels between p45 NFE2 and BACH1 may act as a rheostat favouring either erythroid or MK development.

\subsubsection{SRF and MKL1}

Serum response factor (SRF) is a ubiquitously expressed TF part of the MCM1Agamous-Deficiens-SRF (MADS) family of TFs. SRF binds to CArG sites, the so-called serum response elements, which control more than 150 targets, including genes of the cytoskeleton [155]. Extracellular stimuli can activate SRF through two main signalling pathways: the MAP-kinase pathway through members of the ternary complex factor (TCF) such as ELK1, SAP1 and SAP2 and the small GTPase pathways (Rho family members such as RHO, RAC and CDC42) via two main cofactors from the myocardin-related family, megakaryocytic leukaemia translocation 1 (MKL1, also called MAL) and MKL2 (MAL16). MKL1 was originally described as part of an in-frame translocation $\mathrm{t}(1,22)(\mathrm{p} 13 ; \mathrm{q} 13)$ which fuses MKL1 (encoded on chromosome 22q13) with the protein RBM15 (also called OTT) and is frequently found in acute megakaryoblastic leukaemias occurring de novo in infancy 
or in children older than 1 year, but only occasionally in Down syndrome patients [156-158]. MKL1 activity is regulated by its subcellular localisation. MKL1 binds to monomeric actin (G-actin) which traps it into the cytoplasm. Upon actin polymerisation and formation of F-actin, the pool of G-actin is depleted which promotes MKL1 nuclear localisation [159]. MKL1 expression increases during MK differentiation [160], and its nuclear localisation increases in response to TPO or upon stimulation of the RhoGTPase activation [161, 162].

Srf knockout mice fail to form mesoderm and therefore die early in development. However specific deletion of Srf in the MK compartment using a PF4-Cre system leads to macrothrombocytopenia with accumulation of abnormal low ploidy early MK progenitors. Mkll knockout mice also display thrombocytopenia [160, 163] although with a phenotype that is less severe than the one observed in Srf knockout mice, probably relating to a compensating role of Mkl2 [164].

In both cases, SRF and MKL1 depletion resulted in alterations in cytoskeletal proteins resulting in abnormalities of $\mathrm{MK}$ and platelet ultrastructures and functions. Matrix metalloproteinase 9 (MMP9) and MYL9 are both directly regulated by MKL1, and through dysregulation of the expression of these two proteins, MKL1 deficiency leads to abnormalities of cell migration and proplatelet formation, respectively [161]. MKL1 has also an effect on MK polyploidisation, through dysregulation of RhoA. MK polyploidisation is dependent on RhoA inhibition [165]. RhoA activity is regulated by guanine exchange factors (GEFs) such as ECT2 and GEF-H1. Both of these GEFs are downregulated during MK polyploidisation: the first endomitotic division (from $2 \mathrm{~N}$ to $4 \mathrm{~N}$ ) requires low levels of GEF-H1, whilst subsequent endodivisions require low levels of ECT2. MKL1 null MKs express persistently high levels of GEF-H1 preventing polyploidisation, reflected in the increase in $2 \mathrm{~N}$ immature MKs found in the bone marrow of these mice [166].

Of note, RBM15, the other partner involved in the $\mathrm{t}(1,22)$ megakaryoblastic leukaemias, is an RNA-binding protein, probably involved in transcriptional regulation. RBM15 has been shown to play a role in megakaryopoiesis in its own right. Rbm15 conditional knockout mice show an increase in the myeloid and MK compartment [167]. RBM15 also plays a role in stem cell quiescence by controlling the production of an alternative dominant-negative isoform of the TPO receptor [168].

\subsubsection{NFAT}

The nuclear factor of activated T-cell (NFAT) family of TFs plays a role in a multitude of developmental pathways including in the haematopoietic system. Calcineurin is a calcium-dependent phosphatase which, upon a sustained rise in intracellular calcium, dephosphorylates NFATs, which allows their entry into the nucleus. The two main NFATs expressed in MKs are NFATC1 and NFATC2 [169]. Calcium entry into MKs in response to collagen, for example, has been shown to induce calcineurin/NFATs [170]. It is thought that NFATs negatively regulate megakaryopoiesis in two ways. First is the downregulation of cell cycling and induction of apoptosis. Pharmacological inhibition of calcineurin with cyclosporin A leads to 
thrombocytosis in mice with accumulation of MKs showing an increase in cell cycling driven by enhanced transcription of $C D K 4$ and G1 cyclins [171]. Second, NFATs have been shown to increase expression of FAS ligand (FASLG) on the surface of MKs, increasing apoptosis in the MKs and bystander cells expressing FAS [170].

Interestingly, NFATs may also be implicated in the pro-inflammatory role of platelets. FASLG expression on the surface of platelets has been shown to modulate inflammatory target cells [172]. The regulation of the expression of FASLG on platelets is effected through NFATC2, but modulated by another TF, namely, early growth factor 1 (EGR1) [173]. EGR1 itself has been shown to play a role in megakaryopoiesis including the regulation of the expression of the platelet signal transduction protein $\mathrm{G}_{\alpha q}[174]$.

The NFAT pathway appears to be involved in several MK pathologies. Two genes known to inhibit calcineurin (DSCR1 and DYRK1A) are situated on Chr21 and are overexpressed in Down syndrome, probably contributing to the development of the Down syndrome-associated TMD and AMKL $[175,176]$. This was confirmed in a mouse model where overexpression of a single copy of Dscrl reproduced the results obtained with cyclosporin A inhibition of calcineurin [171]. NFATs are also implicated in myeloproliferative diseases. FKBP5, another calcineurin inhibitor, was found to be upregulated in myelofibrosis [177]. Furthermore, in one patient with essential thrombocythaemia, a three-way translocation which created a breakpoint in NFATC2 was identified possibly contributing to the overproliferation of MKs [178].

\subsubsection{MEIS1 and Its Regulator GFI1B}

Myeloid ecotropic viral integration site 1 (MEIS1) belongs to the TALE class of homeodomain TFs, characterised by three amino acid loop extensions between the $\alpha$-helices of its homeodomain. It interacts with other homeodomain proteins, in particular PBX1 (pre-B-cell leukaemia homeobox 1) forming a DNA-binding heterodimer and cooperatively associates with HOX homeodomain proteins (such as HOXA9). Meis1 knockout mice are embryonically lethal due to impaired vasculogenesis and haematopoiesis alongside limb and eye defects [179].

MEIS1 plays a key role at the stem cell/progenitor stages, promoting appearance of definitive haematopoiesis and cellular self-renewal [180, 181]. However, MEIS1 expression is also maintained specifically in the MK lineage [182]. Overexpression studies have shown a MEIS1-driven induction of a MK transcriptional programme [183] and a skewing towards the MK lineage rather than erythroid development [181]. The embryonic lethality and haemorrhages observed in the MEIS1-deficient animals are attributed to a defect in the separation of lymphatic from the blood vessels secondary to a complete lack in the production of platelets [184].

Further evidence of the role of MEIS1 in the production of platelets was found in a GWAS study which identified the existence of a variant in a binding site for MEIS1 in the gene DNM3 associated with the regulation of platelet count and 
volume [95]. This MEIS1 binding site falls within a region acting as an alternative promoter for DNM3 solely used in MKs [185]. DNM3 has since been confirmed as a key regulator of proplatelet formation [186].

The transcriptional repressor growth factor independence (GFI) has a clearly documented role in maintaining stem cell homeostasis [187], and its homolog GFI1B has been shown to play a role in the emergence of both erythroid and MK lineages in the foetal liver [188]. GFI1B and its cofactors lysine (K)-specific demethylase 1A (LSD1, encoded by the KdmlA gene) and rest corepressor 1 (RCOR1) have been identified in ChIP-Seq studies as key regulators of Meis1 [189]. The evidence that GFI1B plays a role in MK maturation has been recently further exemplified with the identification of a mutation creating a dominant-negative form of GFI1B leading to an autosomal dominant form of gray platelet syndrome [190].

\subsubsection{MYB}

MYB (myeloblastosis viral oncogene homolog, encoded on chromosome 6q22) clearly plays a role in megakaryopoiesis, but essentially at the early progenitor stage. Complete knockdown in the mouse leads to embryonic lethality due to a lack of red cell production. However, in a murine model where the level of MYB activity has been reduced to $10 \%$ of normal rather than abolished, animals can reach adulthood and present with anaemia accompanied by a marked thrombocytosis [191]. Erythroid maturation is impaired due to failure to progress from the BFU-E to erythroblasts stage due to inhibition of the KIT receptor and persistence of "stem cell"-like TFs such as GATA2 and FLI1 [192]. Analysis of the MK compartment, however, shows an increase in the proliferation of early progenitors, but low levels of c-Myb are still permissive of full MK maturation therefore leading to thrombocytosis. Interestingly, in dual Gatallow Myblow mice, the reduction in MYB only partially rescues the thrombocytopenia due to GATA1 deficiency, showing clearly separate, nonoverlapping roles for both TFs [193]. Recently, it was shown that one mechanism through which MYB drives erythroid versus MK differentiation is by the transactivation of miR-486-3p expression resulting in the downregulation of MAF (NFE2 subunit) [194].

\subsubsection{It Is All About Networking}

Although it is clear that the TFs described above play a role in MK differentiation, the level of complexity as to how they regulate gene expression is way beyond their individual function (Fig. 2.2) . Several of the TFs discussed above have been shown to interact with each other to regulate megakaryopoiesis, possibly in a linear hierarchy. For example, GATA1, GATA2 and TAL1 have been shown to regulate NFE2 $[195,196]$. TF biology has to be understood in the context of networks where each TFs will bind with a series of partners that will influence not only its DNA-binding characteristics but also its effect on transcription, i.e. activation or repression as is the case for the regulation of MPL transcription by RUNX1 (see Sect. 2.5.3.3) or the 


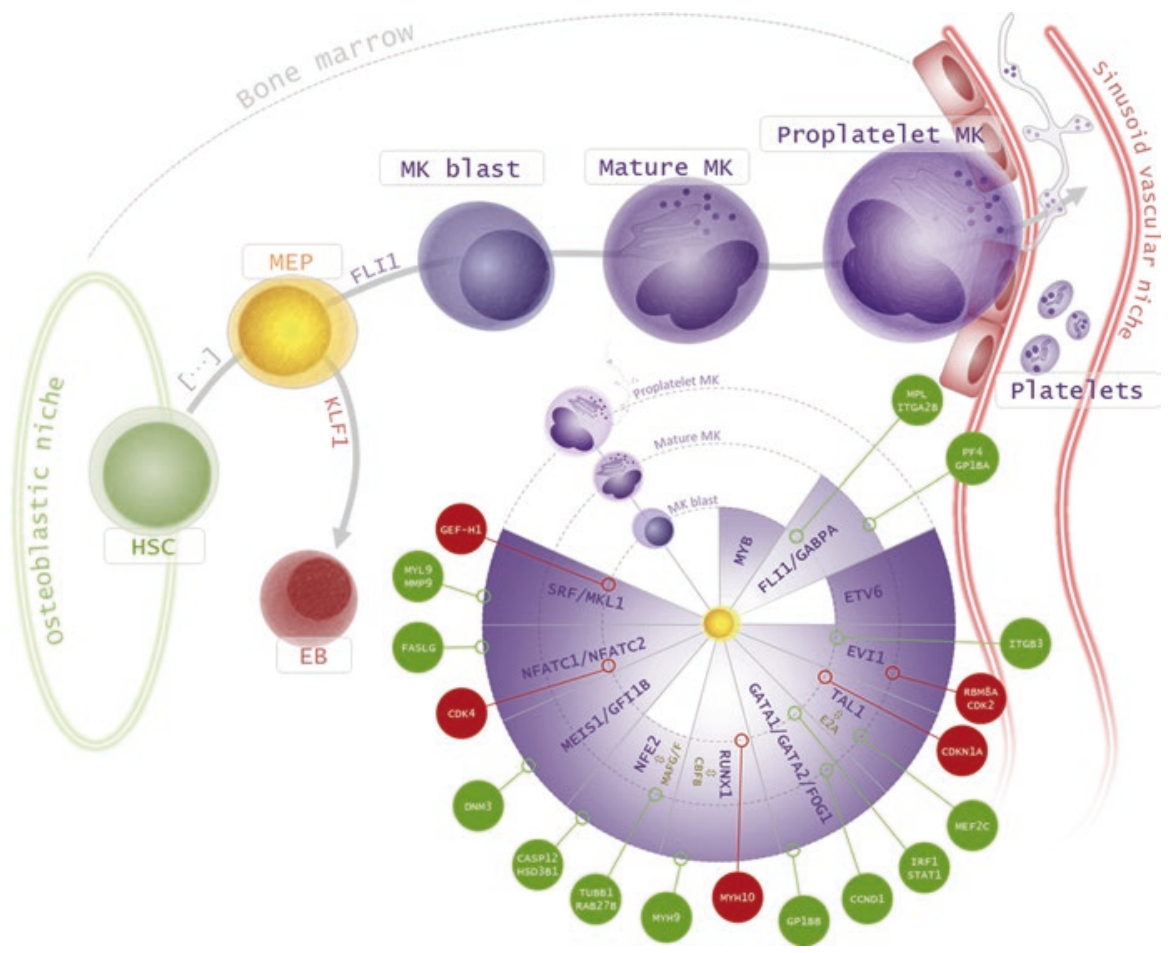

Fig. 2.2 Transcription factors (TFs) and downstream targets regulating megakaryocyte (MK) differentiation. MKs differentiate in the bone marrow from the common erythroid and MK progenitor (MEP), which is derived from the myeloid differentiation arm of the haematopoietic stem cell (HSC). The two TFs KLF1 and FLI1 act in the MEP as master switches to drive erythroid and MK commitment, respectively. Late MK differentiation involves endomitosis, cytoplasmic maturation and proplatelet formation which are accompanied by a progressive journey from the bone marrow to the vascular niche where platelets are released into the circulation upon contact with the endothelial layer of the sinusoids. The MK differentiation wheel depicts the TFs (purple, obligate cofactor in brown) shown to play a role in these processes and the identified downstream targets performing this role (peripheral circles; green and red indicate positive and negative regulation, respectively, by the associated TF). Coloured surfaces represent the differentiation stages the transcription factors have been implicated with. Further details can be found in the main text

selective binding of TAL1 to active GATA1 TF complexes (see Sect. 2.5.2) described above $[107,197]$. In addition, TFs regulate not only expression of other partners with which they cooperate and physically associate but also their own expression levels. Even ubiquitously expressed TFs can regulate proteins specific for a given cell type probably through interactions with cell-type-specific transcription regulators, as a recent study for the ubiquitous TFs SP1 and SP3 indicates. SP1 and SP3 were shown to regulate proteins important for terminal MK maturation and platelet production and function [198]. Several approaches have been taken in order to get a glimpse of how these networks are organised, usually combining cellular data with bespoke statistical tools and in silico algorithms. One group took the approach of looking at 
genome-wide expression arrays in 38 distinct populations of human haematopoietic cells and identified 80 "modules" of highly co-expressed genes. A small number of these modules were used specifically within a certain cell type, but most were "reused" across multiple lineages reflecting functional requirement for certain pathways in various cell states. TFs were also found to belong to certain "modules", some of which were already known to play a regulatory role in the given cell state (e.g. a late erythroid module contains GATA1 and forkhead box O3 [FOXO3]) alongside novel TFs for the specific cell state (nuclear factor I/X [NFIX] and myelin transcription factor 1 [MYT1] in the case in point). In silico analysis of putative TF motifs found in the promoter regions of genes belonging to certain modules combined with expression levels of TFs in each cell state allowed the design of putative hierarchical networks responsible for a cell state and subsequent transition of one cell type to the other [199]. Another group used a more functional approach by directly measuring TF activity using lentiviral vectors where luciferase expression is driven by a TF-dependent responsive element. This approach enables us to build a dynamic picture of several TF activities during differentiation. This particular approach was used in K562 cells that were subsequently differentiated towards the red cell or MK lineage using hemin or TPA (aka PMA), respectively, recording TF activity for GATA1 and GATA2, FLI1, NFE2, MYB, TAL1 and SPI1 (spleen focus forming virus proviral integration oncogene 1, aka PU1). This data was used to build regulatory networks using bespoke software. The results, for example, confirmed the mutually activating interaction between TAL1 and GATA1 [200].

To add to this layer of complexity, just like other proteins, the level of expression of TFs is only one of the multiple facets by which cell fate is controlled. TF activity is also regulated by cell signalling. One of the prime examples of this is the phosphorylation of the STATs and MAP kinases in response to TPO, downstream of MPL and JAK2. The discovery by different groups in 2005 of a JAK2V617F mutation present in $50 \%$ of patients with essential thrombocythaemia (ET) emphasised the importance of the JAK2 signalling pathway in MK growth and the production of platelets [201203]. There is evidence that not only MK growth but also the last steps of maturation are altered in ET. One study showed that culture-derived MKs from patients with ET had an increased ability to form proplatelets and that the number of proplatelet forming MKs in culture correlated with the platelet count in the patient from whom the MKs were derived [204]. This has also been observed in a knock-in Jak2V617F mouse model of ET where higher phosphorylation of STATs and ERK1/2 was demonstrated in bone marrow-derived MKs leading to altered expression profiles [205].

\subsection{Gene Regulation: A Gateway into New Biological Discoveries}

The advent of new generation sequencing and techniques such as ChIP allows us to identify where TFs bind in the genome (Fig. 2.3). As TF binding tends to cluster on sites that are critical for gene regulation, looking at multiple TFs simultaneously greatly improves the ability to infer biological relevance from a binding event. In a 


\section{TF study technologies}

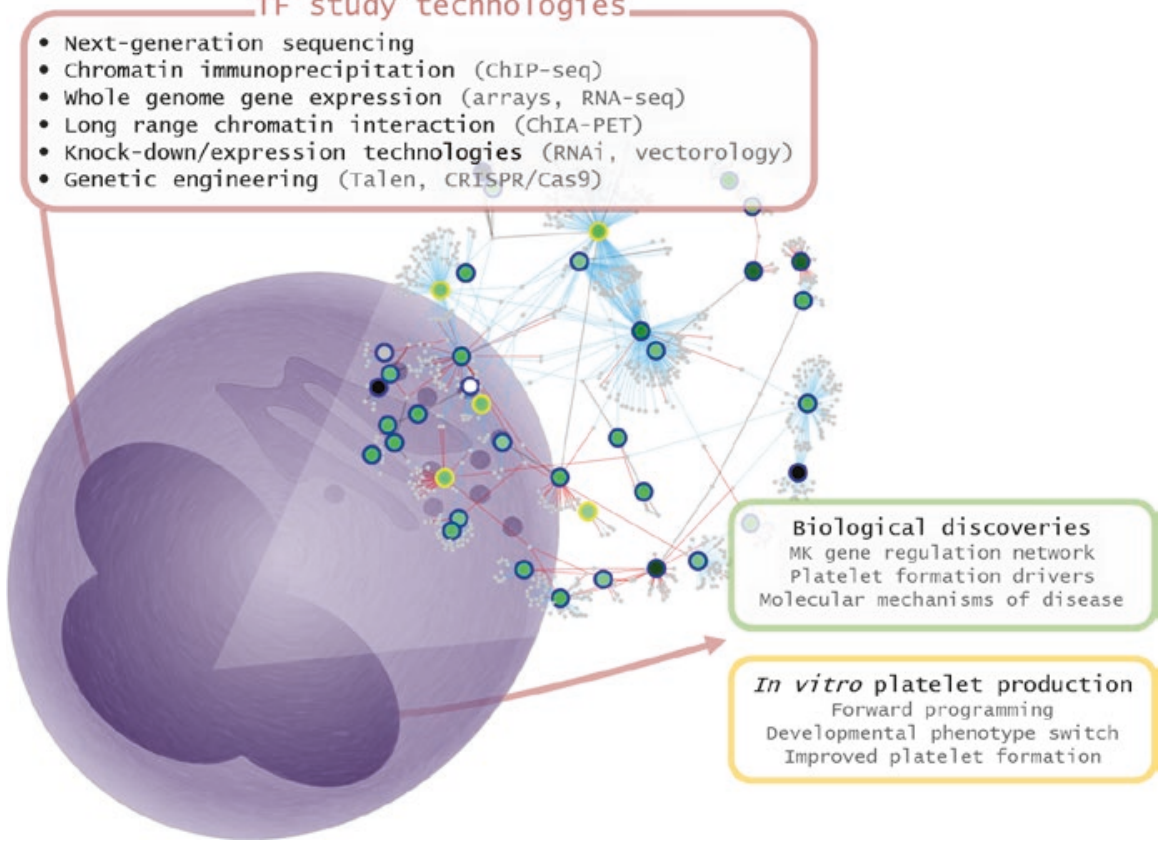

Fig. 2.3 The study of transcription factors (TFs) in megakaryocytes (MKs). The main technologies allowing the deciphering of gene regulatory networks and their control by TFs are indicated. Moving towards a better understanding of molecular mechanisms underlying the MK identity and platelet generation could be ultimately applied to improve in vitro platelet production for clinical development in transfusion medicine

published study, we looked at simultaneous binding of GATA1, GATA2, RUNX1, FLI1 and TAL1 in primary human MKs [206]. In keeping with the existence of TF networks that collaborate to regulate gene expression, simultaneous binding of all five in a given genomic region was particularly enriched, i.e. it occurred far more than would be expected by chance. The five TF-binding sites identified 151 "candidate" genes, some of which are already known to play a key role in megakaryopoiesis, whilst others are not. If one envisions a temporal hierarchy of TFs, it is likely that, amongst the list of genes for which expression is putatively controlled by these five TFs, there are proteins that are crucial in particular for the latter stages of MK maturation, including platelet formation. This is illustrated by the fact that, for seven out of nine genes selected from the 151 targets genes and not previously known to play a role in MKs, there was a clear thrombocyte phenotype upon morpholino knockdown in zebrafish. Just as the genes identified in the study described above are now the subject of ongoing research, regulators of the very late stages of thrombopoiesis, in particular of the proplatelet formation process will potentially be revealed by studies centred on additional genes that are controlled by TFs that have got a clear effect on proplatelet formation (such as NFE2). 
The tumbling costs of sequencing also allows us to look into far more detail at the genetic events that might underlie inherited platelet disorders. In particular, whereas most sequencing studies of pedigrees have in the past focused on exomes, non-coding regions (containing putative regulatory motifs) are now sequenced on an unprecedented scale (www.genomicsengland.co.uk/the-100000-genomesproject). TAR and the GATA-binding-related Bernard-Soulier syndrome may be only two of a series of inherited disorders where the causative mutation lies not in a coding, but in a regulatory region of the genome. Understanding how these genetic variants can lead to disease will further inform our knowledge of how MK maturation is controlled at the transcriptional level.

The same reasoning applies to the understanding of how genetic variations affect gene transcription in healthy individuals. A recent GWAS study identified 68 loci associated with platelet count and volume [95]. Nine of these genes have TF activity, but are completely novel in the context of megakaryopoiesis. These novel factors may regulate genes that affect how platelets are formed.

\subsection{Transcription Factors and the "Making" of Platelets for Clinical Use}

\subsubsection{The Clinical Need for Platelets Produced in the Laboratory}

Thrombocytopenia in patients can be caused by bone marrow failure (inherited and acquired, such as post-cancer treatment) or peripheral bleeding (trauma or surgery), which potentially leads to life-threatening haemorrhages. Treatment relies on transfusion of $\mathrm{ABO}$ and rhesus $\mathrm{D}$-matched platelet concentrates from voluntary donations [207]. The increase in high-dose cancer therapy, advanced surgical procedures and the ageing population has led to a rising demand for platelets with over 4.5 million platelet units transfused per year in Europe and the United States [208]. Platelet transfusion refractoriness in chronically transfused patients and multiparous women necessitates the provision of HLA class 1-matched platelet units, sourced from genotyped recallable donors. The dependence on donations combined with the short shelf life of platelet concentrates (5-7 days) represents a logistical, financial and biosafety challenge for health organisations worldwide.

\subsubsection{Harvesting Platelets from In Vitro-Produced MKs}

Universally the platelet harvest from in vitro-derived MKs has been in single figures [209], whilst MKs in vivo are estimated to produce thousands of platelets per cell $[210,211]$. Maximising platelet production in vitro from these MKs will be a condition without which production of platelets on the scale necessary for a clinically relevant product (each platelet concentrate contains $3 \times 10^{11}$ platelets) will remain a dream. The apparent shortfall in platelet formation might be due to our inability to re-create the niche signal (including shear) necessary for efficient proplatelet 
formation and platelet release, although several approaches using 3D bioreactors have been published to that effect [212-214]. It is also worth mentioning that the evidence that platelets released in vitro are functional has been gathered using descriptive rather than quantitative assays with usually no comparison to platelets freshly isolated from donors. One way to address this issue is to functionally analyse the platelet progeny from in vitro-produced MKs in a mouse transplantation model. Nonobese diabeticsevere combined immunodeficient-IL-2 receptor gamma knockout (NSG) mice completely lack an immune system, which makes them tolerant to human cells [215]. In vitro-grown MKs can be transplanted into irradiated NSG mice and have been shown to produce functional platelets that can be readily detected and functionally analysed by flow cytometry. Although some claim that platelet production happens in the first moments post-MK injection (seemingly in the lung vasculature), the particles detected are clearly heterogeneous, with a significant proportion of "platelets" not expressing the CD42 receptors [216] raising the question whether these particles represent cell debris. We have shown, however, that as from day 3 post-transplantation, a homogeneous population of human platelets appears in the murine peripheral blood, expressing both ITGA2B (CD41) and GP9 (CD42a) and showing the expected response to agonists [217]. Until we have mastered the production of platelets in vitro, the mouse transplantation model probably remains the most reliable way to interrogate the functionality of the platelet progeny from in vitro-cultured MKs.

\subsubsection{Developmental Differences in Megakaryocytes and Platelets: Embryos Are Not Adults}

The development of the haematopoietic system during embryogenesis, the foetal in utero period and after birth has been studied in detail. "Primitive" haematopoiesis first arises in the yolk sack and is followed by the emergence of "definitive" HSCs in the aorta-gonad-mesonephros region, the colonisation of the foetal liver by the haematopoietic system and finally, in the late stage of foetal development, the emergence of the bone marrow as the principal site of haematopoiesis [218]. The evidence that the transition from foetal to adult haematopoiesis is relevant to megakaryopoiesis is exemplified by clinical observations. TMD, characterised by a transient expansion of cells with megakaryoblastic features (see Sect. 2.5.1.3), is seen exclusively in the foetal/neonatal period in some children with Down syndrome. Patients with thrombocytopenia with absent radii (TAR) present in the neonatal period with skeletal abnormalities and low platelet count. The thrombocytopenia associated with TAR however often corrects in the first year of life, although for some patients, the correction can be only partial and occur much later on in life [219].

Understanding the differences in foetal and adult thrombopoiesis has also direct therapeutic implications. The lag time of platelet recovery following cord blood transplantation in comparison to transplantation with stem cells derived from adult donors is much longer. This is a prime example of the difference between neonatal and adult megakaryopoiesis and results in a much longer period of time during which patients are depending on platelet transfusions. 
These differences are also found at the cellular level. It is well known that precursors isolated from the bone marrow have a higher proliferative potential when cultured in vitro for MK production [220, 221]. Also, neonatal MKs are smaller and have a lower ploidy than adult MKs (similar to what is seen for hPSC-derived MKs) [222]. Differences between foetal and adult MKs have also emerged at molecular level which may explain some of the cellular features and clinical findings described above. GATA1 was shown to be overexpressed in CB-MKs compared to adultderived MKs, which also correlated with increased response to TPO through the mTOR pathway [85]. The increase level of GATA1 in foetal MKs might explain the hypersensitivity of neonatal MKs to GATA1 mutations that underlie TMD.

\subsubsection{MKs and Platelets from Human Pluripotent Stem Cells}

Human pluripotent stem cells (hPSCs) can be maintained in vitro whilst retaining the capacity to differentiate towards virtually any cell type upon adequate stimulation [223-225]. The in vitro production of platelets from genetically defined hPSC lines could revolutionise transfusion medicine by providing a controllable source of platelets [226]. Moreover, platelets are anucleate corpuscles that do not proliferate, thus allowing irradiation of the final transfusion product. This represents a marked safety advantage over other hPSC-derived cell therapies where there is an oncogenic risk associated with the chance of administering partially differentiated progenitor cells.

Two types of protocols are in existence to produce MKs from hPSCs. The first relies on external signals provided by cytokines or stromal cells to mimic embryonic development and direct sequential differentiation of hPSCs into MKs, a process designated as "directed differentiation" (DD) [227-231]. These protocols are impaired by a lack of efficiency, low purity of the MKs and often rely on serum and xenogenic feeder cells. Use of serum or xenogenic feeders can present major regulatory issues for the production of a clinical-grade product. The emergence of the knowledge that cell identities can be manipulated by enforcing expression of specific TFs [232] has led investigators to explore the potential of "forward programming" hPSCs towards the MK lineage. Forced expression of GATA1 or TAL1 alone in haematopoietic progenitors has been shown to bias differentiation towards MK and erythroid fates [233, 234]. Accordingly, exogenous expression of TAL1 in human embryonic stem cells has been reported to promote haematopoiesis and megakaryopoiesis [235, 236]. More recently, the combinatorial expression of TAL1 with GATA2 was found to induce a haemogenic endothelial phenotype biased towards erythro-MK differentiation from hPSCs [237]. Proceeding from a methodically curated list of candidate genes, we found that the minimal combination of GATA1, FLI1 and TAL1 results in the highly efficient production of MKs from an array of hPSC lines in chemically-defined serum-free conditions and with minimum cytokine input [238]. None of these three TFs by itself or a combination of two could efficiently induce forward programming and impose MK identity to hPSCs. The proof of the power of the synergistic effect of key transcriptional controllers on cell fate is exemplified by the fact that forward programmed MKs using these three TFs are $>90 \%$ pure, can be cryopreserved, maintained and amplified in vitro for up to 90 days exceeding a 100,000-fold cell yield. Gene expression arrays showed the 
acquisition of a genuine MK phenotype at the whole genome level and notably the appropriate expression of all key MK TFs and effectors discussed above.

Whilst very close phenotypically and at whole genome expression level to MKs derived from primary neonatal haematopoietic progenitors, MKs generated from hPSCs showed a distinct expression signature reminiscent of the "foetal MK profile" regardless of the method of production (directed differentiation or forward programming) [239]. Bluteau et al. showed that this translated into functional differences in particular a reduction in the rate of proplatelet formation in the hPSC-derived MKs. Whether this foetal MK signature has functional consequences on their platelet progeny is unknown. Recent studies in mice showed an extended life span of neonatal platelets and the existence of low ploidy platelet-forming MKs in the early embryo evocative of hPSC-derived MKs [240, 241]. There are also numerous studies on the function of platelets in newborns versus adult platelets (reviewed in [242]) which indicate that differences do exist, although the results are not consistent and the jury is still out as to the clinical significance of these differences. In the future, the manipulation of the TF expression pattern could become a powerful approach to control developmental phenotype switches in MKs produced from different stem cell sources.

\subsubsection{Disease Modelling}

Beyond the production of platelets for clinical use, the development of protocols to generate large amount of MKs from hPSCs has opened new avenues of research in disease modelling. hPSCs can be readily derived from patients' cells that carry specific mutations identified through genetic studies of pedigrees with inherited platelet disorders. The emergence of genome-editing technologies such as transcription activator-like endonucleases (TALENs) and Cas9/clustered regularly interspaced short palindromic repeats (CRISPR) also offers the opportunity to introduce specific mutations in the genome of an hPSC line from which the phenotype and MK output are known using a specific differentiation protocol. The latter approach has the advantage to eliminate potential bias of interpretation due to inter-donor cell line variability [243].

\subsection{Concluding Remarks}

Our knowledge of the key TFs that regulate megakaryopoiesis has grown exponentially over the last 20 years. This has in part been driven by the analyses of the phenotype of murine models where TFs have been knocked down or overexpressed, quite often initially designed to study other cells than MKs (e.g. haematopoietic stem cells). Another important driver has been the advent of sequencing technologies (first Sanger sequencing followed by new generation sequencing), which has allowed us to identify mutations in TFs in pedigrees of human patients with inherited platelet disorders. MKs represent only $0.01 \%$ of the cells in the bone marrow, and it is only since TPO was discovered 20 years ago that we have been able to culture MKs in sufficient quantities to carry out the detailed cellular studies of how these TFs confer cell identity and which specific biological processes they control 
in the maturing MK. More recently, techniques such as ChIP-Seq have allowed us to get a glimpse of the true complexity of transcriptional regulation of the MK, which cannot be understood without putting each individual TF within large regulatory networks that interact with each other in order to confer cellular specificity on gene transcription. It is clear that understanding gene transcription in the MK will be key to unlock how specific cellular processes such as proplatelet formation are controlled which, in turn, will have great bearing on our ability to, at some point in the future, produce MKs and platelets in vitro for clinical use.

Acknowledgements The authors would like to thank Verity Kew for critically reading the text.

\section{References}

1. Doulatov S, Notta F, Laurenti E, Dick JE (2012) Hematopoiesis: a human perspective. Cell Stem Cell 10:120-136

2. Copley MR, Beer PA, Eaves CJ (2012) Hematopoietic stem cell heterogeneity takes center stage. Cell Stem Cell 10:690-697

3. Krause DS, Scadden DT, Preffer FI (2013) The hematopoietic stem cell niche - home for friend and foe? Cytometry B Clin Cytom 84:7-20

4. Sanjuan-Pla A et al (2013) Platelet-biased stem cells reside at the apex of the haematopoietic stem-cell hierarchy. Nature 502:232-236

5. Yamamoto R et al (2013) Clonal analysis unveils self-renewing lineage-restricted progenitors generated directly from hematopoietic stem cells. Cell 154:1112-1126

6. van den Heuvel A, Stadhouders R, Andrieu-Soler C, Grosveld F, Soler E (2015) Long-range gene regulation and novel therapeutic applications. Blood 125:1521-1525

7. Edelstein LC et al (2013) MicroRNAs in platelet production and activation. J Thromb Haemost JTH 11(Suppl 1):340-350

8. Paralkar VR et al (2014) Lineage and species-specific long noncoding RNAs during erythromegakaryocytic development. Blood 123:1927-1937

9. Raslova $\mathrm{H}$ et al (2003) Megakaryocyte polyploidization is associated with a functional gene amplification. Blood 101:541-544

10. Chen L et al (2014) Transcriptional diversity during lineage commitment of human blood progenitors. Science 345:1251033

11. Landry P et al (2009) Existence of a microRNA pathway in anucleate platelets. Nat Struct Mol Biol 16:961-966

12. Denis MM et al (2005) Escaping the nuclear confines: signal-dependent pre-mRNA splicing in anucleate platelets. Cell 122:379-391

13. Lee TI, Young RA (2000) Transcription of eukaryotic protein-coding genes. Annu Rev Genet 34:77-137

14. Ng RK, Gurdon JB (2008) Epigenetic inheritance of cell differentiation status. Cell Cycle Georget Tex 7:1173-1177

15. Cooper G (2000) The cell: a molecular approach. Sinauer Associates, Sunderland

16. Doré LC, Crispino JD (2011) Transcription factor networks in erythroid cell and megakaryocyte development. Blood 118:231-239

17. Gurdon JB (1962) The developmental capacity of nuclei taken from intestinal epithelium cells of feeding tadpoles. J Embryol Exp Morphol 10:622-640

18. Takahashi K, Yamanaka S (2006) Induction of pluripotent stem cells from mouse embryonic and adult fibroblast cultures by defined factors. Cell 126:663-676

19. Geddis AE (2010) Megakaryopoiesis. Semin Hematol 47:212-219

20. Zheng $\mathrm{C}$ et al (2008) TPO-independent megakaryocytopoiesis. Crit Rev Oncol Hematol 65:212-222

21. Malara A, Balduini A (2012) Blood platelet production and morphology. Thromb Res 129:241-244 
22. Avecilla ST et al (2004) Chemokine-mediated interaction of hematopoietic progenitors with the bone marrow vascular niche is required for thrombopoiesis. Nat Med 10:64-71

23. Ito T, Ishida Y, Kashiwagi R, Kuriya S (1996) Recombinant human c-Mpl ligand is not a direct stimulator of proplatelet formation in mature human megakaryocytes. Br J Haematol 94:387-390

24. Choi ES et al (1996) The role of megakaryocyte growth and development factor in terminal stages of thrombopoiesis. Br J Haematol 95:227-233

25. Ogilvy S et al (1999) Constitutive Bcl-2 expression throughout the hematopoietic compartment affects multiple lineages and enhances progenitor cell survival. Proc Natl Acad Sci U S A 96:14943-14948

26. Bouillet $\mathrm{P}$ et al (1999) Proapoptotic Bcl-2 relative Bim required for certain apoptotic responses, leukocyte homeostasis, and to preclude autoimmunity. Science 286:1735-1738

27. Kaluzhny $\mathrm{Y}$ et al (2002) BclxL overexpression in megakaryocytes leads to impaired platelet fragmentation. Blood 100:1670-1678

28. Clarke MCH, Savill J, Jones DB, Noble BS, Brown SB (2003) Compartmentalized megakaryocyte death generates functional platelets committed to caspase-independent death. J Cell Biol 160:577-587

29. De Botton $\mathrm{S}$ et al (2002) Platelet formation is the consequence of caspase activation within megakaryocytes. Blood 100:1310-1317

30. Josefsson EC et al (2011) Megakaryocytes possess a functional intrinsic apoptosis pathway that must be restrained to survive and produce platelets. J Exp Med 208:2017-2031

31. White MJ et al (2012) Caspase-9 mediates the apoptotic death of megakaryocytes and platelets, but is dispensable for their generation and function. Blood 119:4283-4290

32. Debrincat MA et al (2015) BCL-2 is dispensable for thrombopoiesis and platelet survival. Cell Death Dis 6, e1721

33. Hamada $\mathrm{T}$ et al (1998) Transendothelial migration of megakaryocytes in response to stromal cell-derived factor 1 (SDF-1) enhances platelet formation. J Exp Med 188:539-548

34. Avraham H, Cowley S, Chi SY, Jiang S, Groopman JE (1993) Characterization of adhesive interactions between human endothelial cells and megakaryocytes. J Clin Invest 91:2378-2384

35. Pitchford SC, Lodie T, Rankin SM (2012) VEGFR1 stimulates a CXCR4-dependent translocation of megakaryocytes to the vascular niche, enhancing platelet production in mice. Blood 120:2787-2795

36. Hoffman R (1989) Regulation of megakaryocytopoiesis. Blood 74:1196-1212

37. Sabri S et al (2006) Deficiency in the Wiskott-Aldrich protein induces premature proplatelet formation and platelet production in the bone marrow compartment. Blood 108:134-140

38. Mazharian A, Ghevaert C, Zhang L, Massberg S, Watson SP (2011) Dasatinib enhances megakaryocyte differentiation but inhibits platelet formation. Blood 117:5198-5206

39. Sabri S et al (2004) Differential regulation of actin stress fiber assembly and proplatelet formation by alpha2beta1 integrin and GPVI in human megakaryocytes. Blood 104:3117-3125

40. Ghevaert $\mathrm{C}$ et al (2008) A nonsynonymous SNP in the ITGB3 gene disrupts the conserved membrane-proximal cytoplasmic salt bridge in the alphaIIbbeta 3 integrin and cosegregates dominantly with abnormal proplatelet formation and macrothrombocytopenia. Blood 111:3407-3414

41. Bury L, Malara A, Gresele P, Balduini A (2012) Outside-in signalling generated by a constitutively activated integrin $\alpha \operatorname{IIb} \beta 3$ impairs proplatelet formation in human megakaryocytes. PLoS One 7, e34449

42. Balduini A et al (2009) Proplatelet formation in heterozygous Bernard-Soulier syndrome type Bolzano. J Thromb Haemost JTH 7:478-484

43. Golfier S et al (2010) Shaping of terminal megakaryocyte differentiation and proplatelet development by sphingosine-1-phosphate receptor S1P4. FASEB J Off Publ Fed Am Soc Exp Biol 24:4701-4710

44. Zhang L et al (2012) A novel role of sphingosine 1-phosphate receptor S1pr1 in mouse thrombopoiesis. J Exp Med 209:2165-2181

45. Eckly A et al (2014) Biogenesis of the demarcation membrane system (DMS) in megakaryocytes. Blood 123:921-930 
46. Raslova $\mathrm{H}$ et al (2007) Interrelation between polyploidization and megakaryocyte differentiation: a gene profiling approach. Blood 109:3225-3234

47. Chen Z, Hu M, Shivdasani RA (2007) Expression analysis of primary mouse megakaryocyte differentiation and its application in identifying stage-specific molecular markers and a novel transcriptional target of NF-E2. Blood 109:1451-1459

48. Mattia G et al (2002) Different ploidy levels of megakaryocytes generated from peripheral or cord blood CD34+ cells are correlated with different levels of platelet release. Blood 99:888-897

49. Miyazaki R et al (2000) Comparative analyses of megakaryocytes derived from cord blood and bone marrow. Br J Haematol 108:602-609

50. Leysi-Derilou Y, Duchesne C, Garnier A, Pineault N (2012) Single-cell level analysis of megakaryocyte growth and development. Differ Res Biol Divers 83:200-209

51. Jackson CW, Steward SA, Chenaille PJ, Ashmun RA, McDonald TP (1990) An analysis of megakaryocytopoiesis in the $\mathrm{C} 3 \mathrm{H}$ mouse: an animal model whose megakaryocytes have $32 \mathrm{~N}$ as the modal DNA class. Blood 76:690-696

52. Brown AS, Martin JF (1994) The megakaryocyte platelet system and vascular disease. Eur J Clin Invest 24(Suppl 1):9-15

53. Shivdasani RA et al (1995) Transcription factor NF-E2 is required for platelet formation independent of the actions of thrombopoietin/MGDF in megakaryocyte development. Cell 81:695-704

54. Zimmet JM, Toselli P, Ravid K (1998) Cyclin D3 and megakaryocyte development: exploration of a transgenic phenotype. Stem Cells Dayt Ohio 16(Suppl 2):97-106

55. Merika M, Orkin SH (1993) DNA-binding specificity of GATA family transcription factors. Mol Cell Biol 13:3999-4010

56. Tsang AP et al (1997) FOG, a multitype zinc finger protein, acts as a cofactor for transcription factor GATA-1 in erythroid and megakaryocytic differentiation. Cell 90:109-119

57. Trainor CD et al (1996) A palindromic regulatory site within vertebrate GATA-1 promoters requires both zinc fingers of the GATA-1 DNA-binding domain for high-affinity interaction. Mol Cell Biol 16:2238-2247

58. Johnson KD, Kim S-I, Bresnick EH (2006) Differential sensitivities of transcription factor target genes underlie cell type-specific gene expression profiles. Proc Natl Acad Sci U S A 103:15939-15944

59. Zaret KS, Carroll JS (2011) Pioneer transcription factors: establishing competence for gene expression. Genes Dev 25:2227-2241

60. Tsai FY, Orkin SH (1997) Transcription factor GATA-2 is required for proliferation/survival of early hematopoietic cells and mast cell formation, but not for erythroid and myeloid terminal differentiation. Blood 89:3636-3643

61. Wilson NK et al (2010) Combinatorial transcriptional control in blood stem/progenitor cells: genome-wide analysis of ten major transcriptional regulators. Cell Stem Cell 7:532-544

62. Briegel $\mathrm{K}$ et al (1993) Ectopic expression of a conditional GATA-2/estrogen receptor chimera arrests erythroid differentiation in a hormone-dependent manner. Genes Dev 7:1097-1109

63. Pimkin M et al (2014) Divergent functions of hematopoietic transcription factors in lineage priming and differentiation during erythro-megakaryopoiesis. Genome Res 24:1932-1944

64. Chang AN et al (2002) GATA-factor dependence of the multitype zinc-finger protein FOG-1 for its essential role in megakaryopoiesis. Proc Natl Acad Sci U S A 99:9237-9242

65. Huang $\mathrm{Z}$ et al (2009) GATA-2 reinforces megakaryocyte development in the absence of GATA-1. Mol Cell Biol 29:5168-5180

66. Shivdasani RA, Fujiwara Y, McDevitt MA, Orkin SH (1997) A lineage-selective knockout establishes the critical role of transcription factor GATA-1 in megakaryocyte growth and platelet development. EMBO J 16:3965-3973

67. Vyas P et al (1999) Different sequence requirements for expression in erythroid and megakaryocytic cells within a regulatory element upstream of the GATA-1 gene. Dev Camb Engl 126:2799-2811

68. Muntean AG et al (2007) Cyclin D-Cdk4 is regulated by GATA-1 and required for megakaryocyte growth and polyploidization. Blood 109:5199-5207 
69. Ludlow LB et al (1996) Identification of a mutation in a GATA binding site of the platelet glycoprotein Ibbeta promoter resulting in the Bernard-Soulier syndrome. J Biol Chem 271:22076-22080

70. Ciovacco WA, Raskind WH, Kacena MA (2008) Human phenotypes associated with GATA-1 mutations. Gene 427:1-6

71. Freson K et al (2002) Different substitutions at residue D218 of the X-linked transcription factor GATA1 lead to altered clinical severity of macrothrombocytopenia and anemia and are associated with variable skewed X inactivation. Hum Mol Genet 11:147-152

72. Campbell AE, Wilkinson-White L, Mackay JP, Matthews JM, Blobel GA (2013) Analysis of disease-causing GATA1 mutations in murine gene complementation systems. Blood 121:5218-5227

73. Tsang AP, Fujiwara Y, Hom DB, Orkin SH (1998) Failure of megakaryopoiesis and arrested erythropoiesis in mice lacking the GATA-1 transcriptional cofactor FOG. Genes Dev 12:1176-1188

74. Gao Z et al (2010) FOG-1-mediated recruitment of NuRD is required for cell lineage reenforcement during haematopoiesis. EMBO J 29:457-468

75. Miccio A et al (2010) NuRD mediates activating and repressive functions of GATA-1 and FOG-1 during blood development. EMBO J 29:442-456

76. Chou ST et al (2009) Graded repression of PU.1/Sfpi1 gene transcription by GATA factors regulates hematopoietic cell fate. Blood 114:983-994

77. Mancini E et al (2012) FOG-1 and GATA-1 act sequentially to specify definitive megakaryocytic and erythroid progenitors: FOG-1 and GATA-1 in lineage commitment. EMBO J 31:351-365

78. Fox AH et al (1999) Transcriptional cofactors of the FOG family interact with GATA proteins by means of multiple zinc fingers. EMBO J 18:2812-2822

79. Chlon TM, Doré LC, Crispino JD (2012) Cofactor-mediated restriction of GATA-1 chromatin occupancy coordinates lineage-specific gene expression. Mol Cell 47:608-621

80. Hamlett I et al (2008) Characterization of megakaryocyte GATA1-interacting proteins: the corepressor ETO2 and GATA1 interact to regulate terminal megakaryocyte maturation. Blood 112:2738-2749

81. Chou ST et al (2008) Trisomy 21 enhances human fetal erythro-megakaryocytic development. Blood 112:4503-4506

82. Mundschau $\mathrm{G}$ et al (2003) Mutagenesis of GATA1 is an initiating event in Down syndrome leukemogenesis. Blood 101:4298-4300

83. Rainis L et al (2003) Mutations in exon 2 of GATA1 are early events in megakaryocytic malignancies associated with trisomy 21 . Blood 102:981-986

84. Li Z et al (2005) Developmental stage-selective effect of somatically mutated leukemogenic transcription factor GATA1. Nat Genet 37:613-619

85. Klusmann J-H et al (2010) Developmental stage-specific interplay of GATA1 and IGF signaling in fetal megakaryopoiesis and leukemogenesis. Genes Dev 24:1659-1672

86. Kassouf MT et al (2010) Genome-wide identification of TAL1's functional targets: insights into its mechanisms of action in primary erythroid cells. Genome Res 20:1064-1083

87. Wu W et al (2014) Dynamic shifts in occupancy by TAL1 are guided by GATA factors and drive large-scale reprogramming of gene expression during hematopoiesis. Genome Res 24:1945-1962

88. Shivdasani RA, Mayer EL, Orkin SH (1995) Absence of blood formation in mice lacking the T-cell leukaemia oncoprotein tal-1/SCL. Nature 373:432-434

89. Porcher C et al (1996) The T cell leukemia oncoprotein SCL/tal-1 is essential for development of all hematopoietic lineages. Cell 86:47-57

90. Mikkola HKA et al (2003) Haematopoietic stem cells retain long-term repopulating activity and multipotency in the absence of stem-cell leukaemia SCL/tal-1 gene. Nature 421:547-551

91. Schlaeger TM, Mikkola HKA, Gekas C, Helgadottir HB, Orkin SH (2005) Tie2Cre-mediated gene ablation defines the stem-cell leukemia gene (SCL/tal1)-dependent window during hematopoietic stem-cell development. Blood 105:3871-3874

92. Chagraoui $\mathrm{H}$ et al (2011) SCL-mediated regulation of the cell-cycle regulator p21 is critical for murine megakaryopoiesis. Blood 118:723-735 
93. Gekas C et al (2009) Mef2C is a lineage-restricted target of Scl/Tal1 and regulates megakaryopoiesis and B-cell homeostasis. Blood 113:3461-3471

94. Hu X et al (2009) LSD1-mediated epigenetic modification is required for TAL1 function and hematopoiesis. Proc Natl Acad Sci 106:10141-10146

95. Gieger C et al (2011) New gene functions in megakaryopoiesis and platelet formation. Nature 480:201-208

96. Bielczyk-Maczyńska E et al (2014) A loss of function screen of identified genome-wide association study Loci reveals new genes controlling hematopoiesis. PLoS Genet 10, e1004450

97. Liakhovitskaia A et al (2014) Runx1 is required for progression of CD41+ embryonic precursors into HSCs but not prior to this. Dev Camb Engl 141:3319-3323

98. Cai Z et al (2000) Haploinsufficiency of AML1 affects the temporal and spatial generation of hematopoietic stem cells in the mouse embryo. Immunity 13:423-431

99. Ichikawa M et al (2004) AML-1 is required for megakaryocytic maturation and lymphocytic differentiation, but not for maintenance of hematopoietic stem cells in adult hematopoiesis. Nat Med 10:299-304

100. Song WJ et al (1999) Haploinsufficiency of CBFA2 causes familial thrombocytopenia with propensity to develop acute myelogenous leukaemia. Nat Genet 23:166-175

101. Sun L, Gorospe JR, Hoffman EP, Rao AK (2007) Decreased platelet expression of myosin regulatory light chain polypeptide (MYL9) and other genes with platelet dysfunction and CBFA2/RUNX1 mutation: insights from platelet expression profiling. J Thromb Haemost JTH 5:146-154

102. Bluteau D et al (2012) Dysmegakaryopoiesis of FPD/AML pedigrees with constitutional RUNX1 mutations is linked to myosin II deregulated expression. Blood 120:2708-2718

103. Chang Y et al (2007) Proplatelet formation is regulated by the Rho/ROCK pathway. Blood 109:4229-4236

104. Chen Z et al (2007) The May-Hegglin anomaly gene MYH9 is a negative regulator of platelet biogenesis modulated by the Rho-ROCK pathway. Blood 110:171-179

105. Lordier L et al (2012) RUNX1-induced silencing of non-muscle myosin heavy chain IIB contributes to megakaryocyte polyploidization. Nat Commun 3:717

106. Pencovich N, Jaschek R, Tanay A, Groner Y (2011) Dynamic combinatorial interactions of RUNX1 and cooperating partners regulates megakaryocytic differentiation in cell line models. Blood 117:e1-e14

107. Satoh Y et al (2008) AML1/RUNX1 works as a negative regulator of c-Mpl in hematopoietic stem cells. J Biol Chem 283:30045-30056

108. Pencovich $\mathrm{N}$ et al (2013) Cell-autonomous function of Runx1 transcriptionally regulates mouse megakaryocytic maturation. PLoS One 8, e64248

109. Delattre O et al (1992) Gene fusion with an ETS DNA-binding domain caused by chromosome translocation in human tumours. Nature 359:162-165

110. Ben-David Y, Giddens EB, Letwin K, Bernstein A (1991) Erythroleukemia induction by Friend murine leukemia virus: insertional activation of a new member of the ets gene family, Fli-1, closely linked to c-ets-1. Genes Dev 5:908-918

111. Hart A et al (2000) Fli-1 is required for murine vascular and megakaryocytic development and is hemizygously deleted in patients with thrombocytopenia. Immunity 13: $167-177$

112. Athanasiou M et al (1996) Increased expression of the ETS-related transcription factor FLI-1/ ERGB correlates with and can induce the megakaryocytic phenotype. Cell Growth Differ Mol Biol J Am Assoc Cancer Res 7:1525-1534

113. Wang $X$ et al (2002) Control of megakaryocyte-specific gene expression by GATA-1 and FOG-1: role of Ets transcription factors. EMBO J 21:5225-5234

114. Starck J et al (2010) Inducible Fli-1 gene deletion in adult mice modifies several myeloid lineage commitment decisions and accelerates proliferation arrest and terminal erythrocytic differentiation. Blood 116:4795-4805

115. Starck J et al (2003) Functional cross-antagonism between transcription factors FLI-1 and EKLF. Mol Cell Biol 23:1390-1402

116. Pang L et al (2006) Maturation stage-specific regulation of megakaryopoiesis by pointeddomain Ets proteins. Blood 108:2198-2206 
117. Raslova $\mathrm{H}$ et al (2004) FLI1 monoallelic expression combined with its hemizygous loss underlies Paris-Trousseau/Jacobsen thrombopenia. J Clin Invest 114:77-84

118. Favier R et al (2003) Paris-Trousseau syndrome: clinical, hematological, molecular data of ten new cases. Thromb Haemost 90:893-897

119. Loughran SJ et al (2008) The transcription factor Erg is essential for definitive hematopoiesis and the function of adult hematopoietic stem cells. Nat Immunol 9:810-819

120. Carmichael CL et al (2012) Hematopoietic overexpression of the transcription factor Erg induces lymphoid and erythro-megakaryocytic leukemia. Proc Natl Acad Sci U S A 109:15437-15442

121. Goldberg L et al (2013) Genome-scale expression and transcription factor binding profiles reveal therapeutic targets in transgenic ERG myeloid leukemia. Blood 122:2694-2703

122. Birger $Y$ et al (2013) Perturbation of fetal hematopoiesis in a mouse model of Down syndrome's transient myeloproliferative disorder. Blood 122:988-998

123. Stankiewicz MJ, Crispino JD (2009) ETS2 and ERG promote megakaryopoiesis and synergize with alterations in GATA-1 to immortalize hematopoietic progenitor cells. Blood 113:3337-3347

124. Stankiewicz MJ, Crispino JD (2013) AKT collaborates with ERG and Gata1s to dysregulate megakaryopoiesis and promote AMKL. Leukemia 27:1339-1347

125. Wang LC et al (1997) Yolk sac angiogenic defect and intra-embryonic apoptosis in mice lacking the Ets-related factor TEL. EMBO J 16:4374-4383

126. Hock H (2004) Tel/Etv6 is an essential and selective regulator of adult hematopoietic stem cell survival. Genes Dev 18:2336-2341

127. Delwel R, Funabiki T, Kreider BL, Morishita K, Ihle JN (1993) Four of the seven zinc fingers of the Evi-1 myeloid-transforming gene are required for sequence-specific binding to GA(C/T)AAGA(T/C)AAGATAA. Mol Cell Biol 13:4291-4300

128. Kilbey A et al (2005) The Evi1 proto-oncoprotein blocks endomitosis in megakaryocytes by inhibiting sustained cyclin-dependent kinase 2 catalytic activity. Br J Haematol 130:902-911

129. Shimizu S et al (2002) EVI1 is expressed in megakaryocyte cell lineage and enforced expression of EVI1 in UT-7/GM cells induces megakaryocyte differentiation. Biochem Biophys Res Commun 292:609-616

130. Maicas M et al (2012) Functional characterization of the promoter region of the human EVI1 gene in acute myeloid leukemia: RUNX1 and ELK1 directly regulate its transcription. Oncogene. doi:10.1038/onc.2012.222

131. Goyama $S$ et al (2008) Evi-1 is a critical regulator for hematopoietic stem cells and transformed leukemic cells. Cell Stem Cell 3:207-220

132. Yuasa $\mathrm{H}$ et al (2005) Oncogenic transcription factor Evil regulates hematopoietic stem cell proliferation through GATA-2 expression. EMBO J 24:1976-1987

133. Senyuk V et al (2007) Repression of RUNX1 activity by EVI1: a new role of EVI1 in leukemogenesis. Cancer Res 67:5658-5666

134. Albers CA et al (2012) Compound inheritance of a low-frequency regulatory SNP and a rare null mutation in exon-junction complex subunit RBM8A causes TAR syndrome. Nat Genet 44(435-439):S1-S2

135. Letestu $\mathrm{R}$ et al (2000) Existence of a differentiation blockage at the stage of a megakaryocyte precursor in the thrombocytopenia and absent radii (TAR) syndrome. Blood 95:1633-1641

136. Sultan Y, Scrobohaci ML, Rendu F, Caen JP (1972) Abnormal platelet function, population, and survival-time in a boy with congenital absent radii and thrombocytopenia. Lancet 2:653

137. Day HJ, Holmsen H (1972) Platelet adenine nucleotide 'storage pool deficiency' in thrombocytopenic absent radii syndrome. JAMA J Am Med Assoc 221:1053-1054

138. Zahavi J, Gale R, Kakkar VV (1981) Storage pool disease of platelets in an infant with thrombocytopenic absent radii (TAR) syndrome simulating Fanconi's anaemia. Haemostasis 10:121-133

139. Lecine P, Blank V, Shivdasani R (1998) Characterization of the hematopoietic transcription factor NF-E2 in primary murine megakaryocytes. J Biol Chem 273:7572-7578

140. Lecine $P$ et al (1998) Mice lacking transcription factor NF-E2 provide in vivo validation of the proplatelet model of thrombocytopoiesis and show a platelet production defect that is intrinsic to megakaryocytes. Blood 92:1608-1616 
141. Motohashi $\mathrm{H}$ et al (2011) Molecular determinants for small Maf protein control of platelet production. Mol Cell Biol 31:151-162

142. Goerttler PS et al (2005) Gene expression profiling in polycythaemia vera: overexpression of transcription factor NF-E2. Br J Haematol 129:138-150

143. Wang W, Schwemmers S, Hexner EO, Pahl HL (2010) AML1 is overexpressed in patients with myeloproliferative neoplasms and mediates JAK2V617F-independent overexpression of NF-E2. Blood 116:254-266

144. Fock E, Yan F, Pan S, Chong BH (2008) NF-E2-mediated enhancement of megakaryocytic differentiation and platelet production in vitro and in vivo. Exp Hematol 36:78-92

145. Lecine P, Italiano JE Jr, Kim SW, Villeval JL, Shivdasani RA (2000) Hematopoietic-specific beta 1 tubulin participates in a pathway of platelet biogenesis dependent on the transcription factor NF-E2. Blood 96:1366-1373

146. Tiwari S et al (2003) A role for Rab27b in NF-E2-dependent pathways of platelet formation. Blood 102:3970-3979

147. Kerrigan SW, Gaur M, Murphy RP, Shattil SJ, Leavitt AD (2004) Caspase-12: a developmental link between G-protein-coupled receptors and integrin alphaIIbbeta3 activation. Blood 104:1327-1334

148. Nagata $\mathrm{Y}$ et al (2003) Proplatelet formation of megakaryocytes is triggered by autocrinesynthesized estradiol. Genes Dev 17:2864-2869

149. Schwer HD et al (2001) A lineage-restricted and divergent beta-tubulin isoform is essential for the biogenesis, structure and function of blood platelets. Curr Biol CB 11:579-586

150. Kunishima S, Kobayashi R, Itoh TJ, Hamaguchi M, Saito H (2009) Mutation of the beta1tubulin gene associated with congenital macrothrombocytopenia affecting microtubule assembly. Blood 113:458-461

151. Shiraga M et al (1999) Primary megakaryocytes reveal a role for transcription factor NF-E2 in integrin alpha IIb beta 3 signaling. J Cell Biol 147:1419-1430

152. Bord S et al (2004) Estrogen stimulates differentiation of megakaryocytes and modulates their expression of estrogen receptors alpha and beta. J Cell Biochem 92:249-257

153. Fox SW, Chambers TJ (2006) The effect of oestrogen on megakaryocyte differentiation and platelet counts in vivo. Int J Cardiol 109:359-366

154. Toki $\mathrm{T}$ et al (2005) Transgenic expression of BACH1 transcription factor results in megakaryocytic impairment. Blood 105:3100-3108

155. Arsenian S, Weinhold B, Oelgeschläger M, Rüther U, Nordheim A (1998) Serum response factor is essential for mesoderm formation during mouse embryogenesis. EMBO J 17:6289-6299

156. Lion $\mathrm{T}$ et al (1992) The translocation $\mathrm{t}(1 ; 22)(\mathrm{p} 13 ; \mathrm{q} 13)$ is a nonrandom marker specifically associated with acute megakaryocytic leukemia in young children. Blood 79:3325-3330

157. Carroll A et al (1991) The $\mathrm{t}(1 ; 22)$ (p13;q13) is nonrandom and restricted to infants with acute megakaryoblastic leukemia: a Pediatric Oncology Group Study. Blood 78:748-752

158. Bernstein $\mathrm{J}$ et al (2000) Nineteen cases of the $\mathrm{t}(1 ; 22)(\mathrm{p} 13 ; \mathrm{q} 13)$ acute megakaryoblastic leukaemia of infants/children and a review of 39 cases: report from a $t(1 ; 22)$ study group. Leukemia 14:216-218

159. Miralles F, Posern G, Zaromytidou A-I, Treisman R (2003) Actin dynamics control SRF activity by regulation of its coactivator MAL. Cell 113:329-342

160. Cheng E-C et al (2009) Role for MKL1 in megakaryocytic maturation. Blood 113:2826-2834

161. Gilles L et al (2009) MAL/SRF complex is involved in platelet formation and megakaryocyte migration by regulating MYL9 (MLC2) and MMP9. Blood 114:4221-4232

162. Smith EC et al (2013) Induction of megakaryocyte differentiation drives nuclear accumulation and transcriptional function of MKL1 via actin polymerization and RhoA activation. Blood 121:1094-1101

163. Ragu C et al (2010) The serum response factor (SRF)/megakaryocytic acute leukemia (MAL) network participates in megakaryocyte development. Leukemia 24:1227-1230

164. Smith EC et al (2012) MKL1 and MKL2 play redundant and crucial roles in megakaryocyte maturation and platelet formation. Blood 120:2317-2329 
165. Lordier L et al (2008) Megakaryocyte endomitosis is a failure of late cytokinesis related to defects in the contractile ring and Rho/Rock signaling. Blood 112:3164-3174

166. Gao Y et al (2012) Role of RhoA-specific guanine exchange factors in regulation of endomitosis in megakaryocytes. Dev Cell 22:573-584

167. Raffel GD et al (2007) Ott1(Rbm15) has pleiotropic roles in hematopoietic development. Proc Natl Acad Sci U S A 104:6001-6006

168. Xiao $\mathrm{N}$ et al (2015) Ott1 (Rbm15) regulates thrombopoietin response in hematopoietic stem cells through alternative splicing of c-Mpl. Blood 125:941-948

169. Kiani A et al (2007) Expression analysis of nuclear factor of activated T cells (NFAT) during myeloid differentiation of CD34+ cells: regulation of Fas ligand gene expression in megakaryocytes. Exp Hematol 35:757-770

170. Arabanian LS, Kujawski S, Habermann I, Ehninger G, Kiani A (2012) Regulation of fas/fas ligand-mediated apoptosis by nuclear factor of activated $\mathrm{T}$ cells in megakaryocytes. $\mathrm{Br}$ J Haematol 156:523-534

171. Zaslavsky A et al (2013) The calcineurin-NFAT pathway negatively regulates megakaryopoiesis. Blood 121:3205-3215

172. Henn V et al (1998) CD40 ligand on activated platelets triggers an inflammatory reaction of endothelial cells. Nature 391:591-594

173. Crist SA, Elzey BD, Ahmann MT, Ratliff TL (2013) Early growth response-1 (EGR-1) and nuclear factor of activated T cells (NFAT) cooperate to mediate CD40L expression in megakaryocytes and platelets. J Biol Chem 288:33985-33996

174. Jalagadugula G, Dhanasekaran DN, Kim S, Kunapuli SP, Rao AK (2006) Early growth response transcription factor EGR-1 regulates Galphaq gene in megakaryocytic cells. J Thromb Haemost JTH 4:2678-2686

175. Kyttälä S, Habermann I, Minami T, Ehninger G, Kiani A (2009) Regulation of Down syndrome critical region 1 expression by nuclear factor of activated $\mathrm{T}$ cells in megakaryocytes. Br J Haematol 144:395-408

176. Malinge $S$ et al (2012) Increased dosage of the chromosome 21 ortholog Dyrk1a promotes megakaryoblastic leukemia in a murine model of Down syndrome. J Clin Invest 122:948-962

177. Giraudier S et al (2002) Overexpression of FKBP51 in idiopathic myelofibrosis regulates the growth factor independence of megakaryocyte progenitors. Blood 100:2932-2940

178. Vieira L et al (2012) Three-way translocation $(X ; 20 ; 16)(\mathrm{p} 11 ; \mathrm{q} 13 ; \mathrm{q} 23)$ in essential thrombocythemia implicates NFATC2 in dysregulation of CSF2 expression and megakaryocyte proliferation. Genes Chromosomes Cancer 51:1093-1108

179. Hisa $\mathrm{T}$ et al (2004) Hematopoietic, angiogenic and eye defects in Meis1 mutant animals. EMBO J 23:450-459

180. Azcoitia V, Aracil M, Martínez-A C, Torres M (2005) The homeodomain protein Meis1 is essential for definitive hematopoiesis and vascular patterning in the mouse embryo. Dev Biol 280:307-320

181. Cai M et al (2012) Dual actions of Meis1 inhibit erythroid progenitor development and sustain general hematopoietic cell proliferation. Blood 120:335-346

182. Watkins NA et al (2009) A HaemAtlas: characterizing gene expression in differentiated human blood cells. Blood 113:e1-e9

183. Zeddies S et al (2014) MEIS1 regulates early erythroid and megakaryocytic cell fate. Haematologica 99:1555-1564

184. Carramolino L et al (2010) Platelets play an essential role in separating the blood and lymphatic vasculatures during embryonic angiogenesis. Circ Res 106:1197-1201

185. Nürnberg ST et al (2012) A GWAS sequence variant for platelet volume marks an alternative DNM3 promoter in megakaryocytes near a MEIS1 binding site. Blood 120: 4859-4868

186. Wang W, Gilligan DM, Sun S, Wu X, Reems J-A (2011) Distinct functional effects for dynamin 3 during megakaryocytopoiesis. Stem Cells Dev 20:2139-2151

187. Hock $\mathrm{H}$ et al (2004) Gfi-1 restricts proliferation and preserves functional integrity of haematopoietic stem cells. Nature 431:1002-1007 
188. Saleque S, Cameron S, Orkin SH (2002) The zinc-finger proto-oncogene Gfi-1b is essential for development of the erythroid and megakaryocytic lineages. Genes Dev 16:301-306

189. Chowdhury AH et al (2013) Differential transcriptional regulation of meis1 by Gfilb and its co-factors LSD1 and CoREST. PLoS One 8, e53666

190. Monteferrario D et al (2014) A dominant-negative GFI1B mutation in the gray platelet syndrome. N Engl J Med 370:245-253

191. Emambokus N et al (2003) Progression through key stages of haemopoiesis is dependent on distinct threshold levels of c-Myb. EMBO J 22:4478-4488

192. Vegiopoulos A, García P, Emambokus N, Frampton J (2006) Coordination of erythropoiesis by the transcription factor c-Myb. Blood 107:4703-4710

193. García P, Berlanga O, Vegiopoulos A, Vyas P, Frampton J (2011) c-Myb and GATA-1 alternate dominant roles during megakaryocyte differentiation. J Thromb Haemost JTH 9:1572-1581

194. Bianchi E et al (2015) MYB controls erythroid versus megakaryocyte lineage fate decision through the miR-486-3p-mediated downregulation of MAF. Cell Death Differ. doi:10.1038/ cdd. 2015.30

195. Takayama M et al (2010) Genetic analysis of hierarchical regulation for Gata1 and NF-E2 p45 gene expression in megakaryopoiesis. Mol Cell Biol 30:2668-2680

196. McCormack MP et al (2006) A critical role for the transcription factor Scl in platelet production during stress thrombopoiesis. Blood 108:2248-2256

197. Tripic T et al (2009) SCL and associated proteins distinguish active from repressive GATA transcription factor complexes. Blood 113:2191-2201

198. Meinders $\mathrm{M}$ et al (2015) Sp1/Sp3 transcription factors regulate hallmarks of megakaryocyte maturation and platelet formation and function. Blood 125:1957-1967

199. Novershtern N et al (2011) Densely interconnected transcriptional circuits control cell states in human hematopoiesis. Cell 144:296-309

200. Duncan MT et al (2014) Dynamic transcription factor activity profiles reveal key regulatory interactions during megakaryocytic and erythroid differentiation. Biotechnol Bioeng 111:2082-2094

201. Levine RL et al (2005) Activating mutation in the tyrosine kinase JAK2 in polycythemia vera, essential thrombocythemia, and myeloid metaplasia with myelofibrosis. Cancer Cell 7:387-397

202. James $\mathrm{C}$ et al (2005) A unique clonal JAK2 mutation leading to constitutive signalling causes polycythaemia vera. Nature 434:1144-1148

203. Baxter EJ et al (2005) Acquired mutation of the tyrosine kinase JAK2 in human myeloproliferative disorders. Lancet Lond Eng1 365:1054-1061

204. Balduini A et al (2011) In vitro megakaryocyte differentiation and proplatelet formation in $\mathrm{Ph}$-negative classical myeloproliferative neoplasms: distinct patterns in the different clinical phenotypes. PLoS One 6, e21015

205. Hobbs CM et al (2013) JAK2V617F leads to intrinsic changes in platelet formation and reactivity in a knock-in mouse model of essential thrombocythemia. Blood 122:3787-3797

206. Tijssen MR et al (2011) Genome-wide analysis of simultaneous GATA1/2, RUNX1, FLI1, and SCL binding in megakaryocytes identifies hematopoietic regulators. Dev Cell 20:597-609

207. Estcourt LJ et al (2012) Platelet transfusions in haematology patients: are we using them appropriately? Vox Sang 103:284-293

208. Stroncek DF, Rebulla P (2007) Platelet transfusions. Lancet 370:427-438

209. Takayama N et al (2008) Generation of functional platelets from human embryonic stem cells in vitro via ES-sacs, VEGF-promoted structures that concentrate hematopoietic progenitors. Blood 111:5298-5306

210. Harker LA, Finch CA (1969) Thrombokinetics in man. J Clin Invest 48:963-974

211. Kaufman RM, Airo R, Pollack S, Crosby WH (1965) Circulating megakaryocytes and platelet release in the lung. Blood 26:720-731 
212. Nakagawa Y et al (2013) Two differential flows in a bioreactor promoted platelet generation from human pluripotent stem cell-derived megakaryocytes. Exp Hematol 41:742-748

213. Thon JN et al (2014) Platelet bioreactor-on-a-chip. Blood 124:1857-1867

214. Di Buduo CA et al (2015) Programmable 3D silk bone marrow niche for platelet generation ex vivo and modeling of megakaryopoiesis pathologies. Blood 125:2254-2264

215. Shultz LD et al (2005) Human lymphoid and myeloid cell development in NOD/LtSz-scid IL2R gamma null mice engrafted with mobilized human hemopoietic stem cells. J Immunol Baltim Md 1950(174):6477-6489

216. Fuentes $R$ et al (2010) Infusion of mature megakaryocytes into mice yields functional platelets. J Clin Invest 120:3917-3922

217. Tijssen MR et al (2008) Transplantation of human peripheral blood CD34-positive cells in combination with ex vivo generated megakaryocytes results in fast platelet formation in NOD/SCID mice. Leukemia 22:203-208

218. Orkin SH, Zon LI (2008) Hematopoiesis: an evolving paradigm for stem cell biology. Cell 132:631-644

219. Hedberg VA, Lipton JM (1988) Thrombocytopenia with absent radii. A review of 100 cases. Am J Pediatr Hematol Oncol 10:51-64

220. Olson TA, Levine RF, Mazur EM, Wright DG, Salvado AJ (1992) Megakaryocytes and megakaryocyte progenitors in human cord blood. Am J Pediatr Hematol Oncol 14:241-247

221. Liu Z-J et al (2011) Developmental differences in megakaryocytopoiesis are associated with up-regulated TPO signaling through mTOR and elevated GATA-1 levels in neonatal megakaryocytes. Blood 117:4106-4117

222. Ma DC, Sun YH, Chang KZ, Zuo W (1996) Developmental change of megakaryocyte maturation and DNA ploidy in human fetus. Eur J Haematol 57:121-127

223. Thomson JA et al (1998) Embryonic stem cell lines derived from human blastocysts. Science 282:1145-1147

224. Takahashi K et al (2007) Induction of pluripotent stem cells from adult human fibroblasts by defined factors. Cell 131:861-872

225. Murry CE, Keller G (2008) Differentiation of embryonic stem cells to clinically relevant populations: lessons from embryonic development. Cell 132:661-680

226. Takayama N, Eto K (2012) Pluripotent stem cells reveal the developmental biology of human megakaryocytes and provide a source of platelets for clinical application. Cell Mol Life Sci CMLS 69:3419-3428

227. Klimchenko O et al (2009) A common bipotent progenitor generates the erythroid and megakaryocyte lineages in embryonic stem cell-derived primitive hematopoiesis. Blood 114:1506-1517

228. Takayama $\mathrm{N}$ et al (2010) Transient activation of c-MYC expression is critical for efficient platelet generation from human induced pluripotent stem cells. J Exp Med 207:2817-2830

229. Pick M, Azzola L, Osborne E, Stanley EG, Elefanty AG (2013) Generation of megakaryocytic progenitors from human embryonic stem cells in a feeder- and serum-free medium. PLoS One 8, e55530

230. Feng Q et al (2014) Scalable generation of universal platelets from human induced pluripotent stem cells. Stem Cell Rep 3:817-831

231. Gaur M et al (2006) Megakaryocytes derived from human embryonic stem cells: a genetically tractable system to study megakaryocytopoiesis and integrin function. J Thromb Haemost JTH 4:436-442

232. Cherry ABC, Daley GQ (2012) Reprogramming cellular identity for regenerative medicine. Cell 148:1110-1122

233. Iwasaki $\mathrm{H}$ et al (2003) GATA-1 converts lymphoid and myelomonocytic progenitors into the megakaryocyte/erythrocyte lineages. Immunity 19:451-462

234. Valtieri M et al (1998) Enforced TAL-1 expression stimulates primitive, erythroid and megakaryocytic progenitors but blocks the granulopoietic differentiation program. Cancer Res $58: 562-569$ 
235. Real PJ et al (2012) SCL/TAL1 regulates hematopoietic specification from human embryonic stem cells. Mol Ther J Am Soc Gene Ther 20:1443-1453

236. Toscano MG et al (2015) SCL/TAL1-mediated transcriptional network enhances megakaryocytic specification of human embryonic stem cells. Mol Ther J Am Soc Gene Ther 23:158-170

237. Elcheva I et al (2014) Direct induction of haematoendothelial programs in human pluripotent stem cells by transcriptional regulators. Nat Commun 5:4372

238. Moreau T, Evans AL, Vasquez L, Tijssen MR, Yan Y, Trotter MW, Howard D, Colzani M, Arumugam M, Wu WH, Dalby A, Lampela R, Bouet G, Hobbs CM, Pask DC, Payne H, Ponomaryov T, Brill A, Soranzo N, Ouwehand WH, Pedersen RA, Ghevaert C (2016) Largescale production of megakaryocytes from human pluripotent stem cells by chemically defined forward programming. Nat Commun 7:11208.

239. Bluteau $\mathrm{O}$ et al (2013) Developmental changes in human megakaryopoiesis. J Thromb Haemost 11:1730-1741

240. Liu Z-J et al (2014) Expansion of the neonatal platelet mass is achieved via an extension of platelet lifespan. Blood 123:3381-3389

241. Potts KS et al (2014) A lineage of diploid platelet-forming cells precedes polyploid megakaryocyte formation in the mouse embryo. Blood. doi:10.1182/blood-2014-02-559468

242. Ferrer-Marin F, Stanworth S, Josephson C, Sola-Visner M (2013) Distinct differences in platelet production and function between neonates and adults: implications for platelet transfusion practice. Transfusion (Paris) 53:2814-2821; quiz 2813

243. Rouhani F et al (2014) Genetic background drives transcriptional variation in human induced pluripotent stem cells. PLoS Genet 10, e1004432

244. Dumon S et al. (2012) Itga2b regulation at the onset of definitive hematopoiesis and commitment to differentiation. PLoS One 7(8):e43300. doi: 10.1371/journal.pone.0043300

Open Access This chapter is licensed under the terms of the Creative Commons Attribution 4.0 International License (http://creativecommons.org/licenses/by/4.0/), which permits use, sharing, adaptation, distribution and reproduction in any medium or format, as long as you give appropriate credit to the original author(s) and the source, provide a link to the Creative Commons license and indicate if changes were made.

The images or other third party material in this chapter are included in the chapter's Creative Commons license, unless indicated otherwise in a credit line to the material. If material is not included in the chapter's Creative Commons license and your intended use is not permitted by statutory regulation or exceeds the permitted use, you will need to obtain permission directly from the copyright holder. 